ARTICLE

DOI: $10.1038 / s 41467-018-05978-9$

\title{
A molecular switch orchestrates enzyme specificity and secretory granule morphology
}

Suena Ji ${ }^{1}$, Nadine L. Samara ${ }^{2}$, Leslie Revoredo ${ }^{1}$, Liping Zhang ${ }^{1}$, Duy T. Tran ${ }^{2}$, Kayla Muirhead ${ }^{1}$, Lawrence A. Tabak ${ }^{2} \&$ Kelly G. Ten Hagen ${ }^{1}$

Regulated secretion is an essential process where molecules destined for export are directed to membranous secretory granules, where they undergo packaging and maturation. Here, we identify a gene (pgant9) that influences the structure and shape of secretory granules within the Drosophila salivary gland. Loss of pgant9, which encodes an O-glycosyltransferase, results in secretory granules with an irregular, shard-like morphology, and altered glycosylation of cargo. Interestingly, pgant9 undergoes a splicing event that acts as a molecular switch to alter the charge of a loop controlling access to the active site of the enzyme. The splice variant with the negatively charged loop glycosylates the positively charged secretory cargo and rescues secretory granule morphology. Our study highlights a mechanism for dictating substrate specificity within the O-glycosyltransferase enzyme family. Moreover, our in vitro and in vivo studies suggest that the glycosylation status of secretory cargo influences the morphology of maturing secretory granules.

\footnotetext{
${ }^{1}$ Developmental Glycobiology Section, NIDCR, National Institutes of Health, 30 Convent Drive, Bethesda, MD 20892-4370, USA. ${ }^{2}$ Section on Biological Chemistry, NIDCR, National Institutes of Health, 30 Convent Drive, Bethesda, MD 20892-4370, USA. These authors contributed equally: Suena Ji, Nadine L. Samara. Correspondence and requests for materials should be addressed to K.G.T.H. (email: Kelly.Tenhagen@nih.gov)
} 
$\mathrm{R}$ egulated secretion is an essential process in many cells and tissues whereby bioactive molecules are synthesized and stored in membranous secretory vesicles until a trigger signals their release to the extracellular environment ${ }^{1}$. This type of secretion occurs across many organs, including the digestive, reproductive, endocrine, and nervous systems, and provides a means to deliver essential molecules in response to a hormonal or physiological stimulus ${ }^{2,3}$. Defects in the synthesis or appropriate secretion of the cargo can result in diverse diseases, such as diabetes, cystic fibrosis, and inflammatory bowel disorders ${ }^{4-8}$.

The biosynthesis of secreted molecules begins in the endoplasmic reticulum (ER), where proteins are synthesized, folded and then transported to the Golgi apparatus in a COPIIdependent process ${ }^{9}$. Within the Golgi apparatus, a variety of post-translational modifications occur before cargo is packaged into vesicles that bud from the trans-Golgi network (TGN). These immature secretory granules undergo a number of maturation steps including homotypic fusion; acidification of the granular lumen; and proteolytic processing and condensation of the secretory cargo ${ }^{10-16}$. Large, expanded, and highly-glycosylated secretory cargo such as mucins present unique structural challenges and are thought to undergo regulated condensation within the secretory granule as part of the maturation process. For example, the predominant mucin of the mammalian digestive tract, MUC2, undergoes $\mathrm{pH}$ and $\mathrm{Ca}^{2+}$-dependent multimerization and packaging ${ }^{17}$. However, the exact sequence of events and the many factors involved in cargo processing and proper granule maturation remain poorly understood. Likewise, the roles of protein modifications in cargo structure and condensation within the secretory granule remain to be investigated.

The Drosophila salivary gland synthesizes large secretory granules containing high molecular weight mucins that undergo hormone-regulated exocytosis prior to metamorphosis ${ }^{18,19}$. The size of the granules and the fluorescent tools developed by the Drosophila community, including fluorescently-labeled cargo proteins (Sgs3-GFP) ${ }^{20}$, allow one to image granule biogenesis and morphology in detail. Previous studies have shown that secretory granule biogenesis begins at the trans-Golgi network (TGN) where the highly glycosylated secretory cargo is loaded into small, immature granules in a clathrin and AP-1 dependent process ${ }^{21}$. Immature granules undergo a maturation process that remains ill-defined, but involves homotypic fusion ${ }^{14,22}$ that is regulated in part by type II phosphatidylinositol 4-kinase (PI4KII) and the SNARE Snap $24^{23}$. Thus, as development proceeds, many small immature granules $(1 \mu \mathrm{m}$ diameter) fuse to form large, mature granules (3-8 $\mu \mathrm{m}$ diameter), which then await a hormone signal to begin the process of exocytosis. Upon hormone stimulation, mature secretory granules fuse with the apical plasma membrane and release their contents into the salivary gland lumen in a highly organized process that involves membrane mixing, fusion pore formation, and the recruitment of factors involved in linear and branched actin formation ${ }^{24,25}$. Granule collapse and extrusion of the viscous, highly-glycosylated cargo into the salivary gland lumen occurs in an actin and myosin-dependent manner ${ }^{24-26}$. While many factors involved in regulated secretion have been discovered using this system, the factors regulating granule morphology and maturation remain largely unknown.

Here, we use the Drosophila salivary gland to identify a gene (pgant9) affecting secretory granule morphology. Loss of pgant9 results in secretory granules that adopt an irregular, shard-like appearance as they mature. Interestingly, pgant9 encodes an $\mathrm{O}-$ glycosyltransferase that undergoes tissue-specific splicing within the salivary gland to orchestrate a change in enzyme specificity. The salivary gland-specific enzyme variant preferentially glycosylates the positively charged cargo proteins of the salivary gland and rescues granule morphology, suggesting that cargo glycosylation is essential for proper secretory granule morphology and maturation. Additionally, we identify a unique mechanism for altering enzyme specificity by solving the crystal structures of each splice variant. Taken together, our studies elucidate a factor that influences secretory granule morphology and provide evidence that the glycosylation status of cargo may influence granule shape. Moreover, we identify a strategy for modulating substrate specificity among members of this O-glycosyltransferase family.

\section{Results}

pgant9 is required for proper secretory granule formation. Our laboratory has focused on the factors that influence regulated secretion using Drosophila secretory organs ${ }^{14,24,27}$. Drosophila lines carrying a fluorescently-labeled recombinant version of one of the major mucin proteins expressed in the salivary gland (Sgs3$\mathrm{GFP}^{20}$ ), allow one to image secretory granule biogenesis and morphology $20,21,23-25$. Using this system, we find that loss of the conceptual gene CG30463, which was previously identified as a putative member of the UDP-GalNAc:polypeptide $\mathrm{N}$-acetylgalactosaminyltransferase family of enzymes (ppGalNAcTs in mammals and PGANTs in Drosophila) responsible for the Oglycosylation of proteins ${ }^{28-30}$, influences the morphology of mature secretory granules (Fig. 1a). As shown in Fig. 1a, the parental (WT) Sgs3-GFP-containing secretory granules are round and relatively homogeneous in shape, while granules in salivary glands expressing RNAi to CG30463 (CG30463 ${ }^{R N A i}$ ) are highly irregular in size and shape, with an angular or shard-like appearance. Likewise, a deletion that removes exons 1-4 of the CG30463 coding region over a deficiency that removes all of CG30463 (CG30463 ${ }^{\Delta} / D f(2 R)$; Supplementary Fig. 1) also resulted in secretory granules that were irregular in morphology (Fig. 1a). To quantitate differences in granule shape, we performed morphological analysis of circularity of the secretory granules in $W T$, $C G 30463^{R N A i}$ and $C G 30463^{\Delta} / D f(2 R)$ using the Nikon NIS Elements imaging software suite as described in Methods using at least three biological replicates for each genotype. Average circularity values for each genotype are as follows: $\mathrm{WT}=0.834 \pm$ 0.0121; CG30463 ${ }^{R N A i}=0.806 \pm 0.0215$; and $C G 30463^{\Delta} / D f(2 R)=$ $0.745 \pm 0.0126$. $P$-values for WT vs. $C G 30463^{R N A i}$ and WT vs. $C G 30463^{\Delta} / D f(2 R)$ both were calculated to be $<0.001$. Cumulative frequency of circularity was graphed and is shown in Fig. 1b; a clear shift in the overall distribution of the circularity of secretory granules is seen upon knockdown $\left(C G 30463^{R N A i}\right)$ or deletion $\left(C G 30463^{\Delta} / D f(2 R)\right)$ of $C G 30463$ relative to $\mathrm{WT}$, indicating that loss of CG30463 results in aberrant secretory granule morphology.

To identify the stage at which granules adopted an irregular morphology, we next imaged early stages of secretory granule biogenesis. Interestingly, the defects in mature granule morphology seen upon loss of CG30463 (CG30463 $\left.{ }^{\Delta} / D f(2 R)\right)$ were not present in the small, immature granules $(<1 \mu \mathrm{m}$ in diameter $)$ emanating from the Golgi apparatus (Fig. 1c). These immature granules maintained a round, circular shape similar to that seen in WT. However, CG30463-deficient granules developed an irregular morphology at later stages of granule development (>2 $\mu \mathrm{m}$ in diameter) (Fig. 1c). This suggests that loss of CG30463 is affecting some aspects of secretory granule maturation.

To understand how the loss of CG30463 is affecting secretory granule morphology, we next set out to characterize the protein encoded by CG30463. As mentioned previously, CG30463 is predicted to encode a ppGalNAcT/PGANT, which is an Oglycosyltransferase responsible for the post-translational addition of the sugar $N$-acetylgalactosamine (GalNAc) to proteins, a modification typically found on mucins and other secreted and 

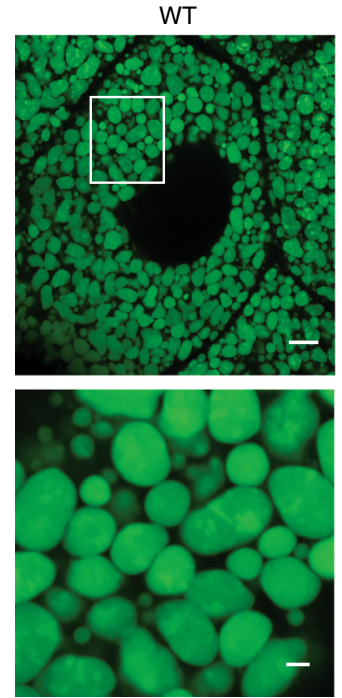

b

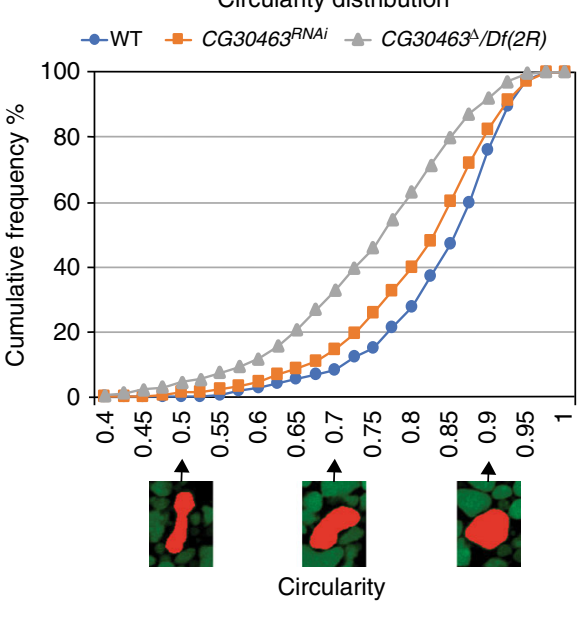

CG30463 RNAi
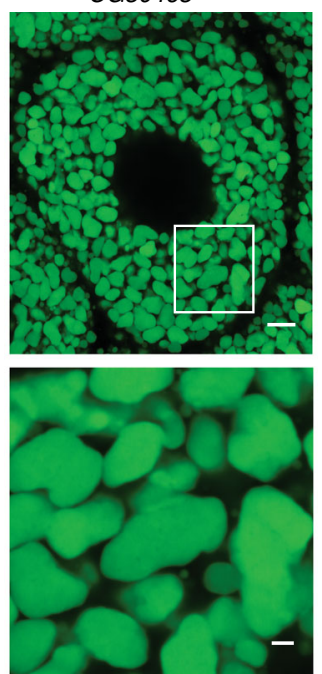

CG30463/ $/ D f(2 R)$
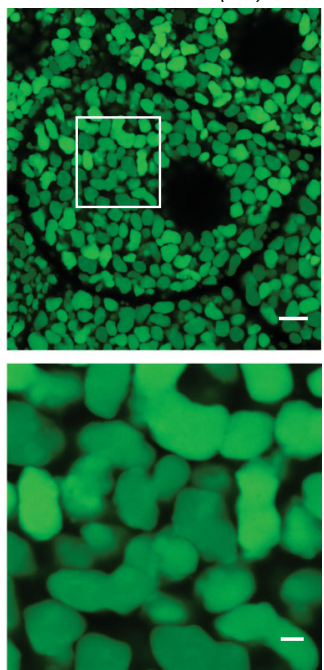

C
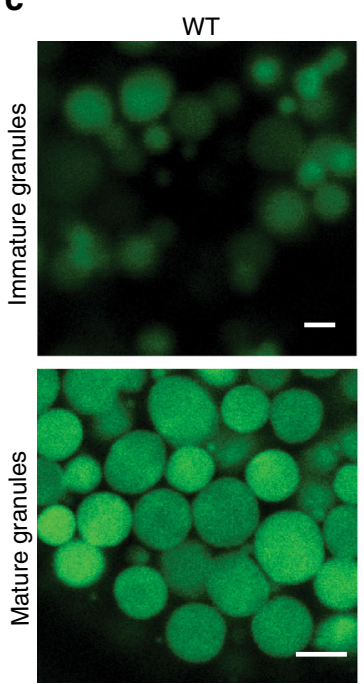

$C G 30463^{\perp} / D f(2 R)$
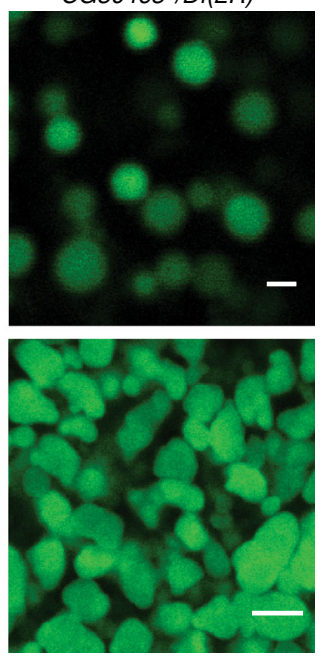

Fig. 1 Irregular secretory granule morphology in the absence of CG30463. a Confocal images of third instar larval salivary glands (SGs) expressing Sgs3GFP cargo (green) in secretory granules. Wild type secretory granules (WT) are round or oval in morphology while granules in SGs expressing RNAi to CG30463 (CG30463 ${ }^{R N A i}$ ) or deficient for CG30463 (CG30463⿻/Df(2R)) are no longer round but have an angular, shard-like morphology. The white boxed area in the upper panels is enlarged below each image. Representative images from three independent experiments are shown. Scale bars, $10 \mu \mathrm{m}$ for the upper panels and $2 \mu \mathrm{m}$ for the lower panels. b Secretory granule circularity for WT, CG30463RNAi, and CG30463 $/ D f(2 R)$ was calculated using the Nikon imaging software. Curves representing cumulative frequency of granule circularity for each genotype are shown. Images of secretory granules corresponding to circularity values of $0.5,0.7$, and 0.9 are shown below the $x$-axis. Granule circularity quantification was performed on granules from three individual salivary glands from each of three independent biological replicates of each genotype. Values were normalized by total number of granules $(n=$ 815 for WT; $n=899$ for CG30463RNAi; $n=841$ for $C G 30463^{4} / D f(2 R)$ ). c Confocal images of secretory granules expressing Sgs3-GFP in proximal (Immature Granules) or distal (Mature Granules) secretory cells of early third instar larval salivary glands from WT and in the absence of CG30463 $\left(C G 30463^{\Delta} / D f(2 R)\right)$. Scale bars, $1 \mu \mathrm{m}$ for the upper panels and $5 \mu \mathrm{m}$ for the lower panels. Representative images from three independent experiments are shown

membrane-bound proteins. ppGalNAcTs are type II membrane proteins that belong to the CAZy family GT2 $7^{31}$. These retaining glycosyltransferases, which catalyze the transfer of GalNAc from UDP-GalNAc to threonine or serine residues within protein substrates, are anchored to the Golgi membrane via a single pass transmembrane domain, and contain a luminal portion that consists of a stem region; a GT-A type catalytic domain with a $\mathrm{Mn}^{2+}$ ion coordinated by a DHH motif ${ }^{32,33}$; and a C-terminal lectin domain, which adopts a $\beta$-trefoil fold (belonging to the carbohydrate-binding module (CBM) group 13 in the CAZy database $\left.^{31}\right)$. Interestingly, we found two distinct Drosophila cDNA clones emanating from the CG30463 gene, differing only in a $\sim 30$ amino acid region (the a subunit) of the lectin domain (Fig. 2a). The lectin domain has been shown to bind extant GalNAc on previously glycosylated substrates to position the catalytic domain further away for additional GalNAc transfer ${ }^{34,35}$. Comparison of these clones to genomic sequence data revealed that they represent splice variants of exon 8 (Fig. 2a). This splicing event generates an $\alpha$ subunit with a net positive charge (isoform $\mathrm{A}$, containing exon $8 \mathrm{~A}$ ) or a net negative charge (isoform B, containing exon 8B) (Fig. 2a). When expressed as full length proteins in Drosophila S2R + cells, both isoforms localized to the Golgi apparatus (Fig. 2b) and generated an increase in O-glycosylation as detected by the lectin HPA (Helix 
a

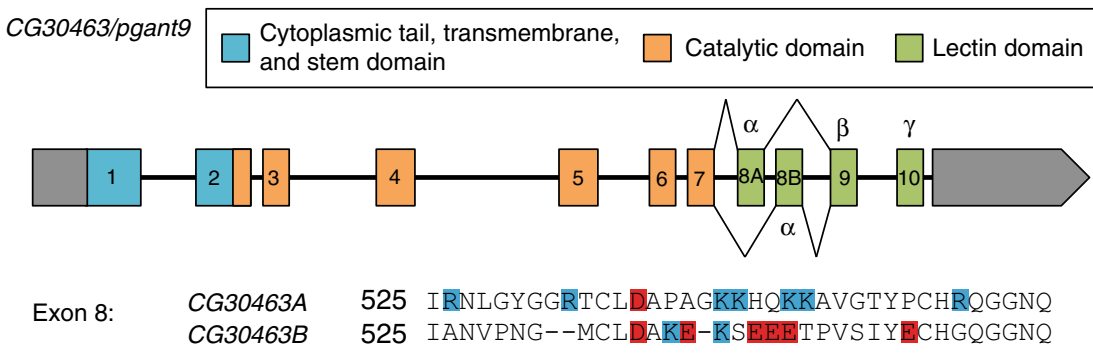

b

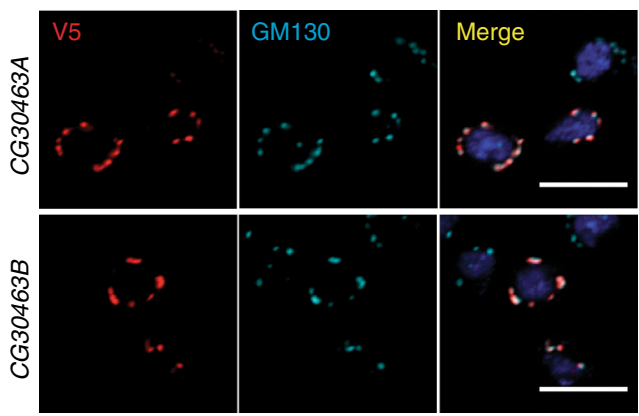

C

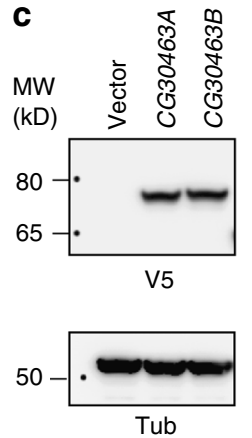

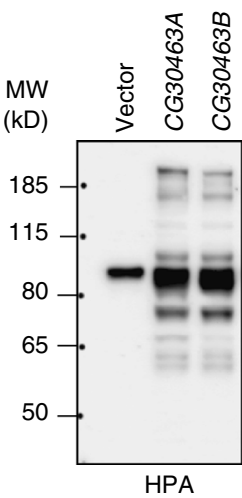

Fig. 2 CG30463/pgant9 encodes a Golgi-localized O-glycosyltransferase. a Gene structure for CG30463/pgant9 is shown, with boxes representing exons and lines representing introns. The $\mathrm{N}$-terminal (blue), catalytic (orange) and lectin (green) domains of the putative glycosyltransferase encoded by CG30463 are shown. The lectin domain consists of three subdomains ( $\alpha, \beta$, and $\gamma$ ). The sequence for the differentially spliced $\alpha$ subdomain (exon 8 ) is shown, with acidic residues highlighted in red and basic residues highlighted in blue. b Both splice variants (V5-tagged; red) localized to the Golgi apparatus (as detected by anti-GM130; blue) in S2R+ cells. Scale bar, $10 \mu \mathrm{m}$. Representative images from two independent experiments are shown. c Western blots of S2R+ cells expressing vector alone (Vector), a V5-tagged recombinant CG30463A or a V5-tagged recombinant CG30463B. Panels on the left show CG30463A and CG30463B expression with the V5-tag (anti-V5) and loading controls (anti-tubulin). Panel on the right shows increased Oglycosylation (as detected by the lectin HPA) when CG30463A or CG30463B are expressed in S2R+ cells. Representative western blots from three independent experiments are shown. Molecular weight markers $(k D)$ are shown to the left of each panel

a

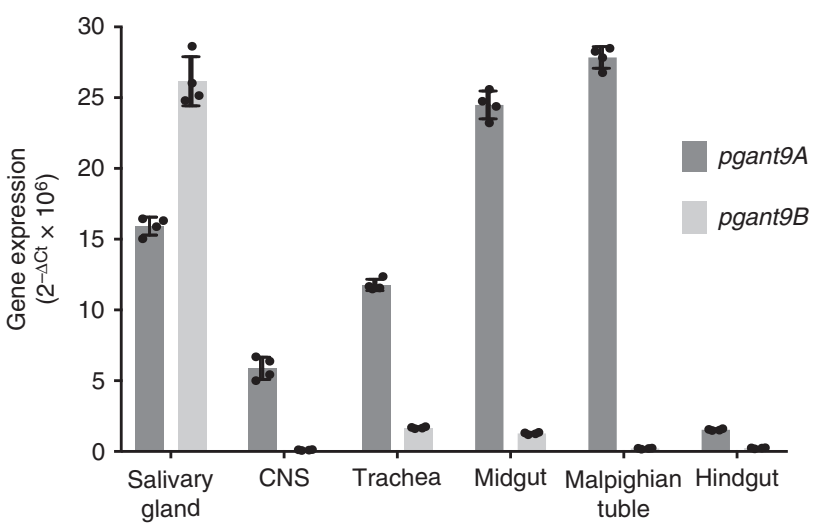

b

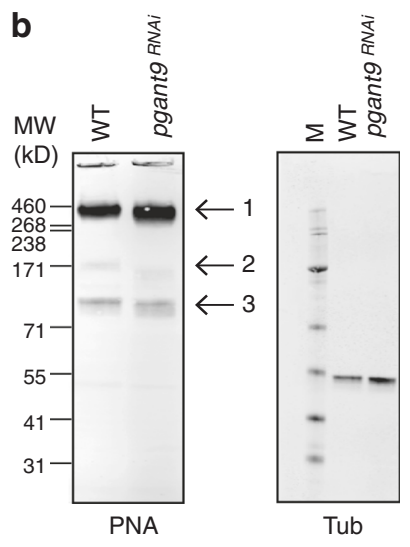

Fig. 3 pgant9 undergoes tissue-specific splicing. a Expression of each splice variant was quantitated in various larval tissues by qPCR. pgant9B expression is most abundant in the salivary gland while pgant9A is the predominant isoform in other tissues examined. RNA levels were normalized to $18 \mathrm{~S} r R N A$. Values represent mean \pm s.d. from four experiments. b Western blots of salivary gland extracts from WT larvae or larvae expressing RNAi to pgant9 (pgant9RNAi) probed with a lectin (peanut agglutinin; PNA) that detects the major salivary gland O-glycans. Size shifts in the three major PNA-reactive bands are seen (denoted with arrows on the right side of the panel). Tubulin loading control is shown in the lower panel. Representative western blot from four independent experiments are shown. Size markers are shown to the left of each panel

pomatia agglutinin, which detects proteins modified with $\mathrm{O}$ linked GalNAc) (Fig. 2c), suggesting that both encode functional, Golgi-localized O-glycosyltransferases. We hereafter refer to the gene CG30463 as pgant9 and designate the splice variants as pgant9A and pgant9B and the enzyme variants they encode as PGANT9A and PGANT9B.
PGANT9A and B differentially glycosylate cargo proteins. To investigate how pgant9 splicing is regulated in vivo, we examined expression levels of each splice variant in larval tissues (Fig. 3a). pgant9A was the predominant form in most tissues (including the trachea, midgut, hindgut, CNS and Malpighian tubules) with the exception of the salivary gland. pgant $9 B$ was the predominant 
splice variant present in the larval salivary glands, suggesting a possible unique requirement for this isoform in that tissue.

To begin to identify the substrates of PGANT9A and PGANT9B in salivary glands, we performed western blotting and mass spectrometry on $W T$ and $C G 30463^{R N A i}$ salivary glands. Westerns probed with a lectin (peanut agglutinin; PNA) that recognizes O-glycosylated proteins $(\mathrm{Gal} \beta 1,3 \mathrm{GalNAc-O}-\mathrm{S} / \mathrm{T})^{36}$ revealed mobility shifts in the three major PNA reactive bands upon knockdown of pgant9 in salivary glands, suggesting changes in the O-glycosylation status of these proteins (Fig. 3b). Mass spectrometry identified one of the major components of bands 1 and 3 as the salivary gland mucin Sgs3 (Supplementary Tables 1 and 2). While band 3 likely represents a glycosylated version of an Sgs 3 monomer or dimer, band 1 runs much higher than the predicted molecular weight of glycosylated Sgs3. This suggests that a population of Sgs3 exists as a high molecular weight multimer, possibly through bonding via cysteines present in the $\mathrm{N}$-terminal and C-terminal regions, similar to what is seen for other mucins. The major component of band 2 was previously identified as the protein Eig71Ee (encoded by CG7604), another protein secreted from the salivary gland that has been shown to be $\mathrm{O}$-glycosylated ${ }^{37}$. Interestingly, the repetitive/O-glycosylated regions of both Eig71Ee and Sgs3 are unique in that they contain positively charged amino acid residues amongst the threonine and serine residues predicted to be O-glycosylated.

To investigate whether Sgs3 is glycosylated by PGANT9A or PGANT9B, we co-expressed full length Sgs3 in Drosophila S2R+ cells with either PGANT9A or PGANT9B (Fig. 4 and Supplementary Fig. 2a). While both PGANT9A and PGANT9B were able to glycosylate Sgs3 (as detected by increased HPA reactivity and an increase in size), the size shift was reproducibly greater in the presence of PGANT9B, suggesting that PGANT9B confers increased glycosylation (Fig. 4b). Additionally, there did not appear to be an additive effect when both PGANT9A and PGANT9B were present together (Supplementary Fig. 2b). To investigate this further, we created deletion constructs of Sgs3 that eliminated one or both of the regions predicted to be glycosylated, and co-expressed them with either PGANT9A or PGANT9B. The first region contains stretches of threonines and is designated the threonine-rich (T-rich) region; the second region is comprised predominantly of repeating units of the sequence PTTTK and is designated the PTTTK region (Fig. $4 \mathrm{a}$ and Supplementary Fig. 2a). Deletion of both regions $(\operatorname{Sgs} 3-\Delta)$ resulted in no glycosylation by either PGANT9A or PGANT9B (Fig. 4c). Interestingly, while constructs containing only the $\mathrm{T}$-rich region (Sgs3-T-rich) were glycosylated similarly (as shown by a similar size shift in the HPA-reactive material) in the presence of either PGANT9A or PGANT9B (Fig. 4d), constructs containing only the PTTTK region (Sgs3-PTTTK) showed a reproducible increase in the size of the HPA-reactive material in the presence of PGANT9B relative to PGANT9A, suggesting that this region is preferentially glycosylated by PGANT9B (Fig. 4e). Taken together, these data suggest that the T-rich region of Sgs 3 is an in vivo substrate for both PGANT9A and PGANT9B, but PGANT9B preferentially acts on the positively-charged PTTTK repetitive region.

Splicing of pgant9 generates a unique charged loop. To understand how the lectin domain- $\alpha$ subunit influences enzymatic specificity, X-ray crystal structures of PGANT9A and PGANT9B in the presence of UDP, $\mathrm{Mn}^{2+}$, and an Sgs3 peptide substrate were solved at atomic resolution (Fig. 5; Supplementary Fig. 3; Table 1). PGANT9A and PGANT9B adopt the canonical ppGalNAcT tertiary structure where a $\sim 10$ amino acid flexible linker connects the $\mathrm{N}$-terminal catalytic domain to the $\mathrm{C}$-terminal lectin domain (Fig. 5a, b) ${ }^{38-42}$. PGANT9A crystallizes with 4 molecules in the asymmetric unit, and each molecule contains electron density in the active site consistent with a catalytic $\mathrm{Mn}^{2+}$ ion coordinated by the conserved active site residues Asp 301, His 303 , and His 437, and 2 oxygens from the $\alpha$ and $\beta$ phosphate of UDP (Fig. 5a). However, there is insufficient electron density to model in a peptide, and the partially ordered active site loop containing residues Arg 440 to Arg 448 is in a semi-closed conformation $^{43}$. PGANT9B crystallizes with 2 molecules per asymmetric unit, but density for UDP, $\mathrm{Mn}^{2+}$, or a peptide is not present in the active site under these crystallization conditions. The active site residue Asp 301 is misaligned, and the active site loop is disordered (Fig. 5b).

PGANT9A and PGANT9B are superimposable, showing that differences in the a subunit between the 2 enzymes do not impact the orientation of the lectin domain relative to the catalytic domain and that the enzymatic specificity is exclusively sequence dependent (Fig. 5c). The a subunit, shown in dark blue in PGANT9A and magenta in PGANT9B, contains a charged loop (Fig. 5a, b, respectively). In PGANT9A, Lys 542, Lys 543, Lys 546, and Lys 547 in the a subunit loop form a basic patch that extends towards the active site with the shortest distance of $\sim 14 \AA$ from Lys 542 to the nearest UDP phosphate (Figs. 5a, 6a). Arg 526, Arg 533, and Arg 556 form a distinct basic patch at the opposite end of the a subunit (Figs. 5a, 6a). The distribution of positively charged residues in the a subunit of PGANT9A creates a predominantly positively charged patch that extends the entire length of the lectin domain (Figs. 5a, c, 6a), and the proximity of the positively charged loop to the active site suggests that it dictates preferences for substrates that contain negatively charged residues, as the positively charged surface could form non-specific electrostatic interactions with the substrate to position it for catalysis. Likewise, positively charged substrates might be excluded from accessing the active site given the position of the positively charged loop. The variable loop in PGANT9B adopts a similar conformation as the loop in PGANT9A (Fig. 5b, c), except that negatively charged residues Glu 538, Glu 541, Glu 542, and Glu 543 are clustered at the region of the loop near the active site to form a negatively charged patch (Figs. 5b, 6b). Another charged region is evident in the electrostatic potential map, where residues including Asp 535 and Glu 550 form a distinct negatively charged patch on the surface of the lectin domain (Figs. 5b, 6b).

Although the charged patches on the surface of the lectin domain consisting of Arg 526, Arg 533, and Arg 556 in PGANT9A and Asp 535 and Glu 550 in PGANT9B are distant from the active site, peptide substrates that are greater than $\sim 16$ amino acids can extend the entire length of the lectin domain as shown in the structure of ppGalNAc-T2 bound to Muc5AC ${ }^{41}$, supporting an additional role of the base of the a-subunit in potential substrate binding. Moreover, the peptides represent a fragment of what are usually much larger protein substrates that could potentially make contacts with various parts of the enzyme. Thus, both enzymes have two distinct charged patches in their lectin domain formed by the a-subunit that results in preferences to recruit either negatively (PGANT9A) or positively (PGANT9B) charged substrates to the active site for catalysis (Fig. 6a, b).

We wondered if the orientation of the a subunits in PGANT9A and PGANT9B were unique compared with other members of the ppGalNAcT family whose structures have been solved. While the a subunit is a highly variable region among ppGalNAcT isoforms (Fig. 6c), the overall fold of the a subunit is conserved. As shown by previous structures of ppGalNAc-T1, ppGalNAc$\mathrm{T} 2$, and ppGalNAc-T10 ${ }^{38-41}$, the orientation of the a subunit varies significantly among the isoforms (Fig. 6d). In PGANT9A 

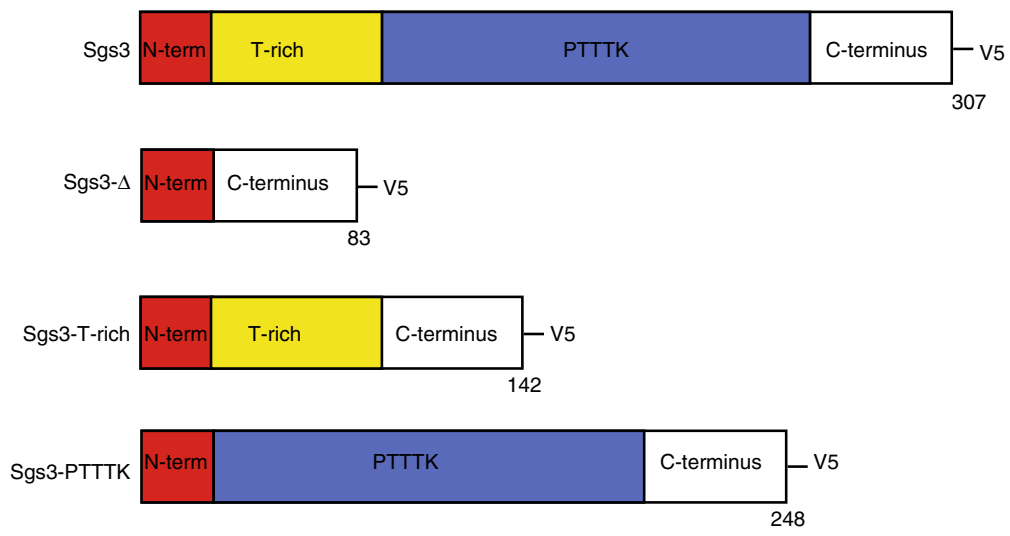

b

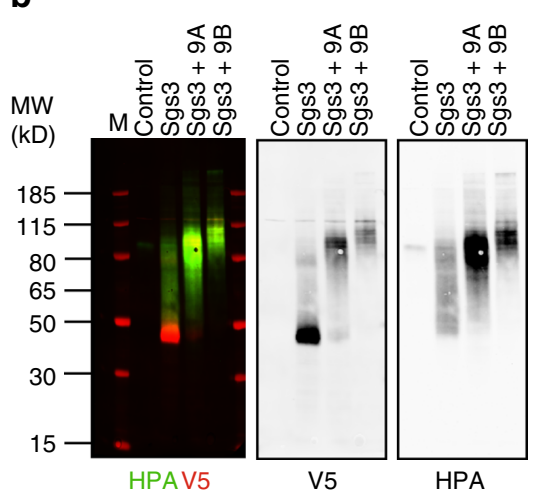

d

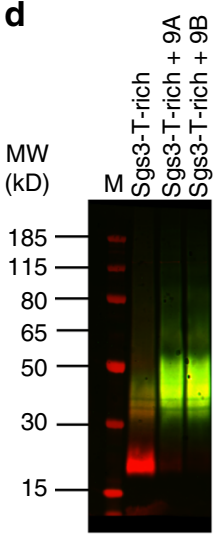

HPAV5

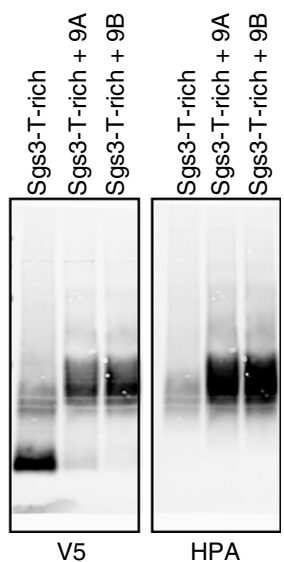

C

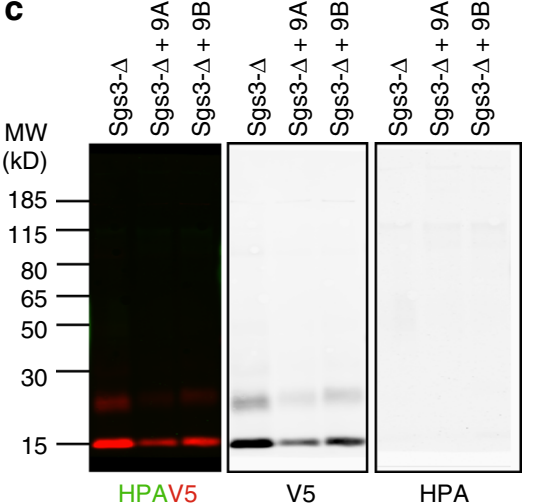

e

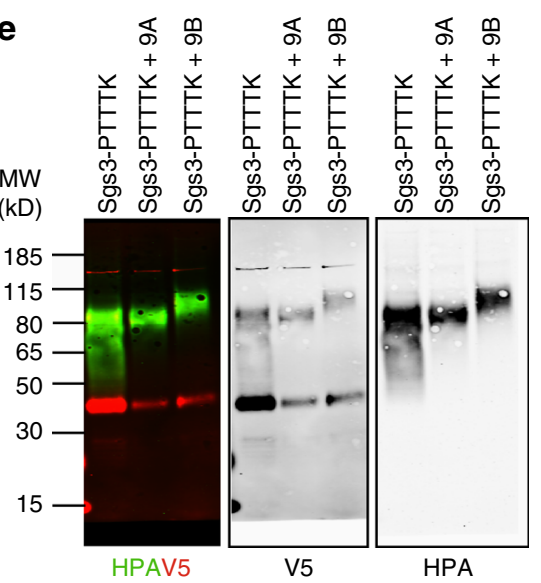

Fig. 4 Differential glycosylation of Sgs3 by PGANT9A and PGANT9B. a Diagrams of full length Sgs3 and Sgs3 deletion constructs are shown (sequences are shown in Supplementary Fig. 2a). The $\mathrm{N}$-terminal region is shown in red and the two distinct regions predicted to be glycosylated are shown in yellow (T-rich) and blue (PTTTK). The C-terminal region is shown in white. All constructs are V5-tagged. $\mathbf{b}$ Western blots of purified proteins from S2R+ cells transfected with empty vector (control), the full length Sgs3 construct (Sgs3), Sgs3 with a plasmid expressing PGANT9A (Sgs3 + 9A) or Sgs3 with a plasmid expressing PGANT9B (Sgs3 + 9B). Westerns were probed to detect the Sgs3 protein (V5; red) and changes in glycosylation (HPA; green). Westerns of purified proteins from similar transfections using the Sgs3- $\Delta$ construct (c), the Sgs3-T-rich construct (d), and the Sgs3-PTTTK construct (e) are also shown. Representative images from three independent experiments are shown. Size markers are shown to the left of each panel

and PGANT9B, the loop is closer to the active site than in the other ppGalNAcTs. In ppGalNAc-T1, which is the human homolog most like PGANT9, the a subunit is positioned further from the active site and the loop appears to be less extended (Fig. 6d). The a subunit of ppGalNAc-T10 also adopts a conformation similar to those of PGANT9A/B, but is shifted away from the active site when the catalytic domains of PGANT9A/B and ppGalNAc-T10 are aligned. Alignment of the catalytic domains of PGANT9A/B and ppGalNAc-T2 results in the misalignment of their lectin domains, where the ppGalNAc-
T2 a subunit is positioned in the opposite orientation of the PGANT9A/B a subunit. As a result, the loop in the a subunit is furthest away from the active site in ppGalNAc-T2. Thus, the a subunit in PGANT9A/B is unique to both enzymes compared with the other isoforms in both its orientation and proximity to the active site. The region is also highly charged compared with other isoforms, which suggests that PGANT9 distinctively evolved to recognize highly charged substrates via its lectin domain and adopted splicing of the a subunit to alter that charge based on the needs of the cell/tissue. 
a

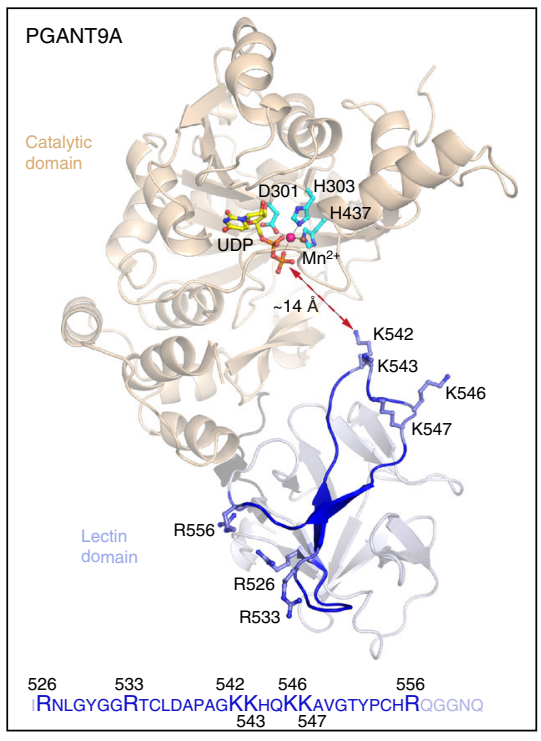

b

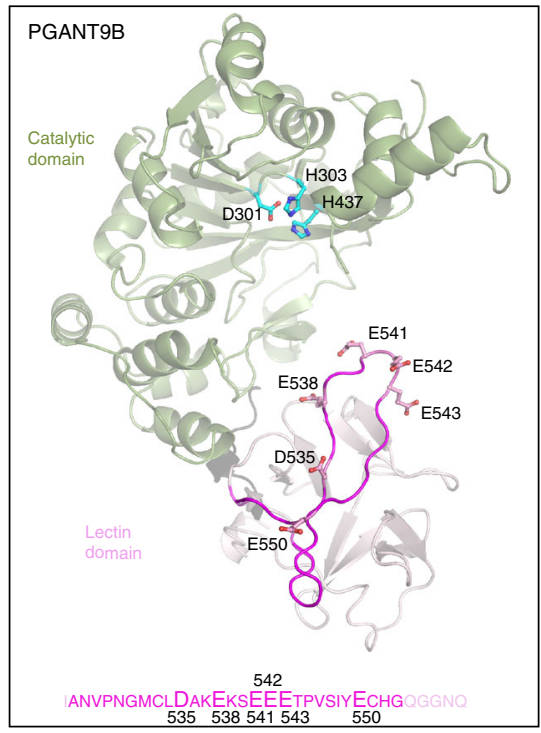

C

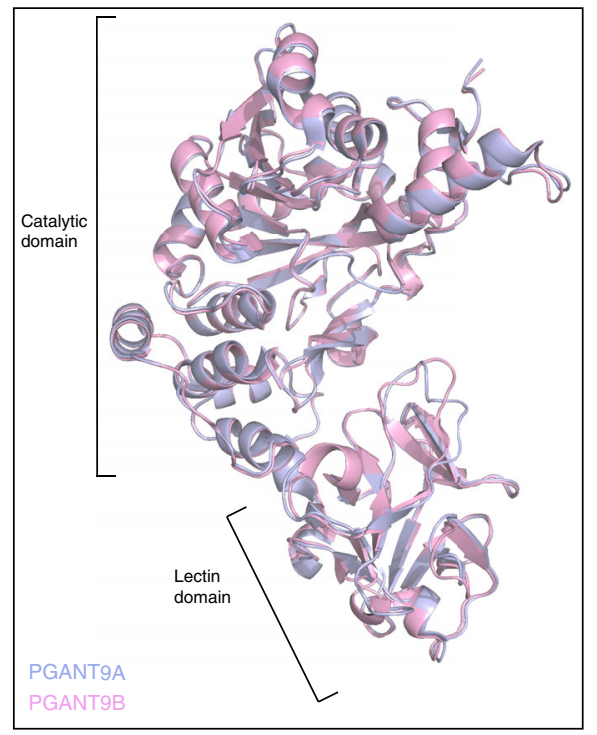

Fig. 5 Crystal structures of PGANT9A and PGANT9B reveal divergent substrate preferences. a Crystal structure of PGANT9A with the catalytic domain shown in wheat and the lectin domain shown in light blue. The active site residues D301, H303, and H437 are in cyan, Mn2+ in magenta, and UDP in yellow. The variable $\alpha$ subunit is in dark blue, and the positively charged residues are shown as sticks. The variable region sequence is in dark blue and the residues shown in the structure are in larger font. The conserved residues on the peripheries are shown in light blue. b Crystal structure of PGANT9B with the catalytic domain in green, the lectin domain in pink, and the $\alpha$ subunit in magenta (with negatively charged residues shown as sticks). The variable region sequence is in magenta and the negatively charged residues are in larger font, while the conserved residues on the peripheries are in light pink. c PGANT9A and PGANT9B adopt the same overall fold. PGANT9A is in light blue, and PGANT9B is in pink

Table 1 Data collection and refinement statistics

\begin{tabular}{|c|c|c|}
\hline & $\begin{array}{l}\text { PGANT9A }^{b} \\
\text { PDB 6E4Q }\end{array}$ & $\begin{array}{l}\text { PGANT9Bb } \\
\text { PDB 6E4R }\end{array}$ \\
\hline \multicolumn{3}{|l|}{ Data collection } \\
\hline $\begin{array}{l}\text { Space group } \\
\text { Cell dimensions }\end{array}$ & 1121 & 1121 \\
\hline 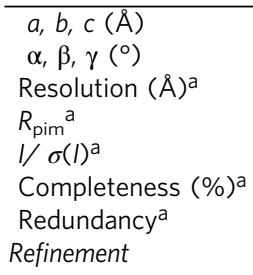 & $\begin{array}{l}126.3,168.8,153.1 \\
90,106.3,90 \\
20.0-2.80(2.90-2.80) \\
0.125(0.583) \\
6.00(1.00) \\
94.1(70.0) \\
6.8(3.5)\end{array}$ & $\begin{array}{l}113.9,48.4,232.0 \\
90,91.4,90 \\
20.0-2.06(2.14-2.06) \\
0.081(0.660) \\
8.64(1.03) \\
96.7(83.4) \\
3.2(3.0)\end{array}$ \\
\hline $\begin{array}{l}\text { Resolution } \\
\text { No. reflections } \\
R_{\text {work }} / R_{\text {free }} \\
\text { No. atoms }\end{array}$ & $\begin{array}{l}19.95-2.80 \\
70,993 \\
19.0 / 26.0\end{array}$ & $\begin{array}{l}20.0-2.06 \\
76,400 \\
16.4 / 20.7\end{array}$ \\
\hline $\begin{array}{l}\text { Protein } \\
\mathrm{Mn}^{2+} \\
\text { UDP/GIcNAc } \\
\text { Water/Solvent } \\
\text { B-factors }\left(\AA^{2}\right)\end{array}$ & $\begin{array}{l}16,242 \\
4 \\
100 / 56 \\
194 / 36\end{array}$ & $\begin{array}{l}8096 \\
0 \\
0 / 28 \\
711 / 86\end{array}$ \\
\hline $\begin{array}{l}\text { Average } \\
\text { Macromolecules } \\
\text { Solvent }{ }^{\mathrm{c}} \\
\text { Ligands } \\
\text { R.m.s deviations }\end{array}$ & $\begin{array}{l}53.6 \\
53.6 \\
35.9 \\
67.9\end{array}$ & $\begin{array}{l}37.2 \\
36.6 \\
40.8 \\
57.5\end{array}$ \\
\hline $\begin{array}{l}\text { Bond lengths }(\AA) \\
\text { Bond angles }\left({ }^{\circ}\right)\end{array}$ & $\begin{array}{l}0.009 \\
1.16\end{array}$ & $\begin{array}{l}0.007 \\
0.99\end{array}$ \\
\hline $\begin{array}{l}\text { bData from a single cryst } \\
\text { cWater, (poly)ethylene g }\end{array}$ & $\begin{array}{l}\text { highest-resolution shell } \\
\text { e used for solving the structure } \\
\text { and glycerol }\end{array}$ & \\
\hline
\end{tabular}

The charged loop controls peptide substrate specificity. To conclusively demonstrate the ability of the charged a subunit loop to dictate specificity toward charged substrates, we performed in vitro enzymatic assays with purified PGANT9A and PGANT9B, as described previously ${ }^{29}$. Putative substrate peptides were designed based on the predicted regions of glycosylation within Sgs3 (Fig. 7a). In agreement with what was seen in cell culture (Fig. 4), in vitro enzyme assays revealed that both PGANT9A and PGANT9B are capable of glycosylating the neutral T-rich peptide derived from the $\mathrm{T}$-rich region (PPTQQSTTQPPCTTS), but only PGANT9B was able to efficiently glycosylate the positively charged PTTTK peptide derived from the PTTTK repetitive region (PTTTKPTTTKPTTTK) (Fig. 7b). To further interrogate this model, we redesigned the PTTTK peptide to replace all positively charged amino acids with negatively charged ones (PTTTE peptide; PTTTEPTTTEPTTTE) (Fig. 7a). As predicted, PGANT9A (with its positively charged a subunit loop) was able to efficiently glycosylate the negatively charged PTTTE peptide while PGANT9B displayed little activity (Fig. 7b). Taken together, our data support a model where tissuespecific splicing of the a subunit of the lectin domain creates a unique charged loop that influences access to the active site, thereby regulating the ability of PGANT9A and PGANT9B to modify highly charged substrates. This provides an example of both splicing altering the substrate specificity of a member of this enzyme family and changes within the lectin domain influencing preferences for unglycosylated substrates.

To address the isoform-specific roles of PGANT9A and PGANT9B in secretory granule morphology, we next performed rescue experiments with each individual isoform. Overexpression of each splice variant was verified at both the RNA and protein levels (Supplementary Fig. 4). Interestingly, overexpression of only pgant $9 B$ in salivary glands deficient for both isoforms (pgant9 ${ }^{R N A i}$, pgant $9 B^{O E}$ ) resulted in the rescue of secretory granule morphology and circularity (Fig. 7c, d). Overexpression of pgant9A only (pgant9 ${ }^{R N A i}$, pgant9A ${ }^{O E}$ ) did not rescue granule 
a

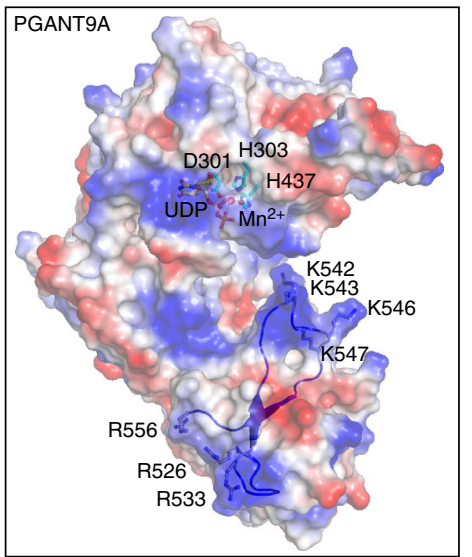

C

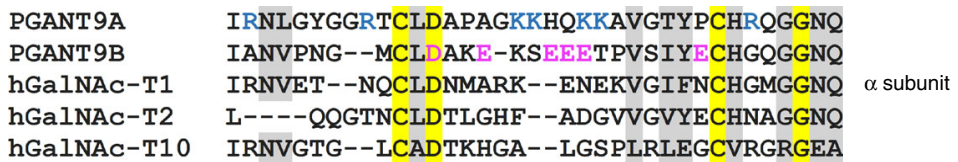

b

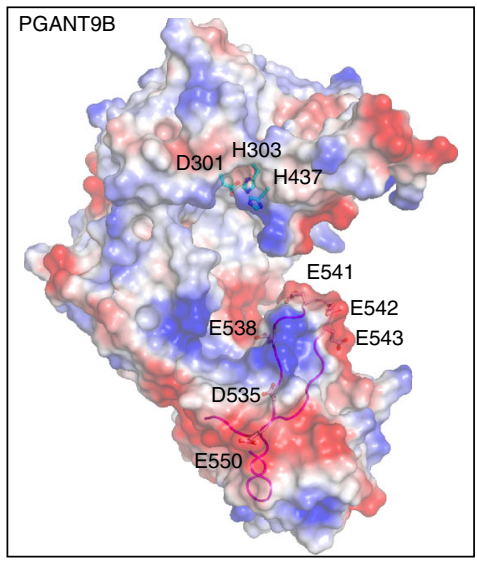

d

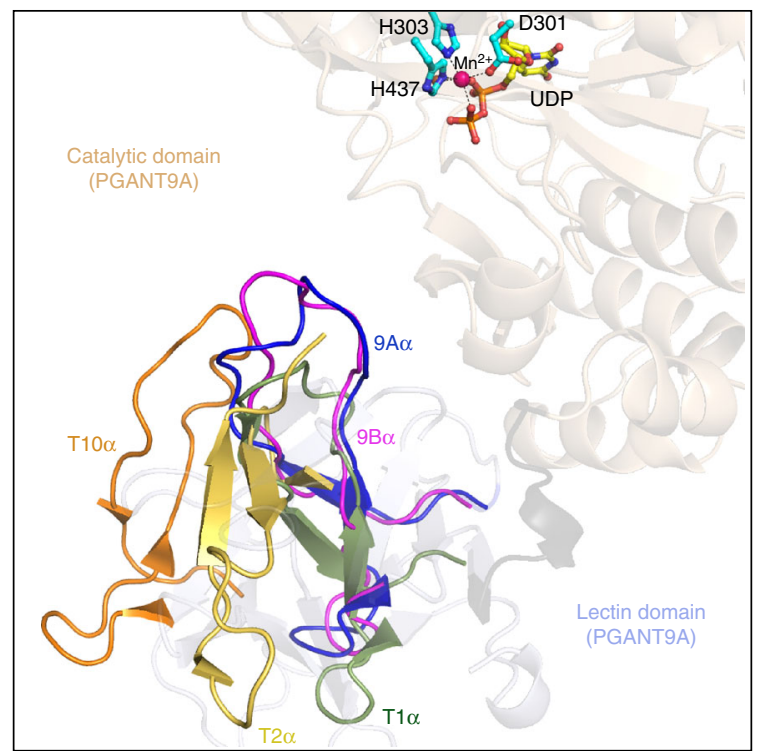

Fig. 6 The $\alpha$ subunits of PGANT9A and PGANT9B determine their unique substrate specificities. a Electrostatic potential surface map of PGANT9A, where the blue regions are positively charged, and red regions are negatively charged superposed over the variable loop shown in dark blue. Basic residues in the loop are shown as sticks. b Electrostatic potential surface map of PGANT9B, where the blue regions are positively charged, and red regions are negatively charged superposed over the variable loop shown in magenta. Acidic residues in the loop are shown as sticks. c Sequence alignment of the $\alpha$ subunit of various isoforms. The $\alpha$ subunit is poorly conserved. Conserved residues are highlighted in yellow, and moderately conserved residues are highlighted in gray. The basic residues in PGANT9A are blue and the acidic residues in PGANT9B are in magenta. $\mathbf{d}$ The $\alpha$ subunits adopt distinct conformations. The $\alpha$ subunits of PGANT9A colored blue, PGANT9B colored magenta, ppGalNAc-T1 colored green, ppGalNAc-T2 colored yellow and ppGalNAc-T10 colored orange are superposed over the crystal structure of PGANT9A with the catalytic domain colored wheat, and the lectin domain colored light blue

morphology/circularity (Fig. 7c, d). Average circularity values for each genotype are as follows: pgant $9^{R N A i}$, pgant $9 A^{O E}=0.798 \pm$ $0.00555 ; \quad$ pgant $9^{R N A i}, \quad$ pgant $9 B^{O E}=0.837 \pm 0.0125$. Taken together, our results provide evidence that differential splicing of an O-glycosyltransferase can alter substrate specificity by generating a charged loop that lies in proximity to the active site, regulating preferences for charged substrates. Moreover, our in vitro and in vivo studies suggest that the glycosylation status of secretory cargo influences the morphology of mature secretory granules.

\section{Discussion}

Here we identify a factor that affects secretory granule morphology. Upon loss of pgant9, granules adopt an irregular shardlike appearance during maturation. Moreover, our in vitro and in vivo data indicate that the enzymatic product of pgant9 specifically glycosylates the secretory cargo produced by the salivary gland, suggesting that defects in cargo glycosylation are influencing the maturation and final morphology of secretory granules. Glycosylation of proteins is known to influence their structure $^{44-46}$. In particular, the high levels of O-glycosylation typically seen in the serine and threonine-rich repetitive regions of mucins are known to confer extended, rod-like structures to these regions ${ }^{44,45,47}$. MUC2, a highly O-glycosylated intestinal mucin, has been shown to dimerize via a C-terminal cysteine knot domain, and then trimerize via $\mathrm{N}$-terminal von Willebrand domains in a $\mathrm{pH}$ and $\mathrm{Ca}^{2+}$-dependent manner ${ }^{17,48,49}$. The internal highly glycosylated mucin domains are thought to assemble into parallel rods during this process, allowing the $\mathrm{N}$ terminal domains to interact and form the concatenated ring structures seen in secretory granules ${ }^{17}$. One can thus imagine how loss of glycosylation and collapse of the rod-like structure could interfere with the orderly packing and multimerization of other regions during granule maturation, thereby changing the structure of the packaged cargo and potentially influencing the shape of the secretory granule. A model where cargo glycosylation affects the packaging that occurs during granule maturation fits nicely with our data showing that early, immature secretory granules have a normal appearance but as granule fusion and maturation proceeds, abnormal morphologies develop. However, it remains possible that PGANT9B may have other substrates within the salivary gland that could also be contributing to changes in granule morphology. Ongoing studies are aimed at imaging secretory granules and their cargo via electron microscopy to determine how proteins are packaged as maturation proceeds in wild type granules and how that packaging may be altered in the absence of PGANT9B.

In this study, we also elucidate a mechanism that regulates enzyme specificity and substrate selectivity within the ppGalNAcT/PGANT family. From previous structural studies, we know that the ppGalNAcT enzymes consist of a catalytic domain and a tripartite lectin domain that are separated by a flexible linker region. Based on previous structural and biochemical studies, the lectin domain was thought to only recognize GalNAc sugars on previously glycosylated substrates, as illustrated in structures of ppGalNAc-T2 and ppGalNAc-T4 bound to glycosylated substrates, where the substrate GalNAc is bound to the lectin domain via a conserved sugar binding pocket in the a subunit ${ }^{41,42}$; this recognition then served to position the catalytic domain near unmodified residues so that additional GalNAcs can be added a certain distance away from the extant $\operatorname{sugar}^{34,35,38,39,41,42}$. No prior evidence existed for the lectin domain influencing the glycosylation of unglycosylated (unmodified) substrates. Indeed, previously solved crystal structures for members of this enzyme family show that the a subunit of the lectin domain is not close 
a

\begin{tabular}{ll}
\hline Peptide & Sequence \\
\hline T-rich & PPTQQSTTQPPCTTS \\
PTTTK & PTTTKPTTTKPTTTK \\
PTTTE & PTTTEPTTTEPTTTE \\
\hline
\end{tabular}

C
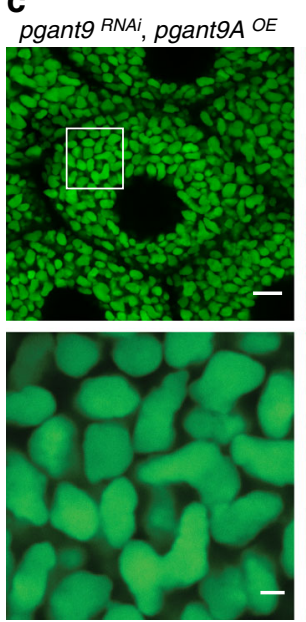

pgant9 ${ }^{R N A i}$, pgant9B $O E$
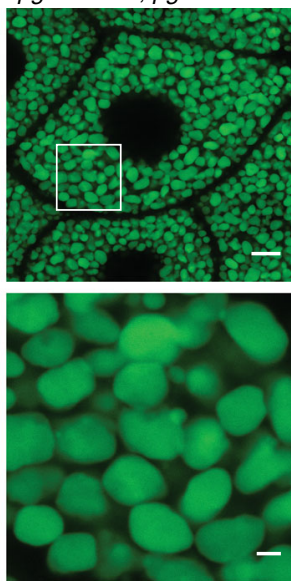

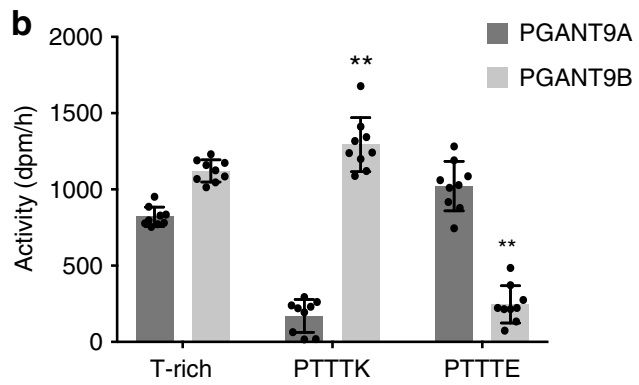

d

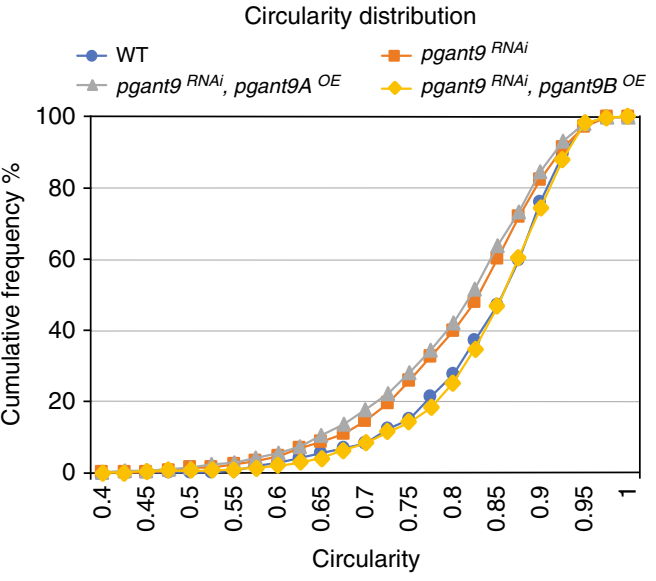

Fig. 7 PGANT9B glycosylates Sgs3 and rescues secretory granule morphology. a Peptides used for in vitro reactions. T-rich is a peptide within the T-rich domain of Sgs3. PTTTK is a peptide within the positively-charged PTTTK repetitive region of Sgs3. PTTTE is a version of PTTTK where all positivelycharged amino acids (K; lysine) have been replaced with negatively-charged amino acids (E; glutamic acid). $\mathbf{b}$ In vitro enzymatic assays measuring the glycosyltransferase activity of purified PGANT9A and PGANT9B against peptides derived from Sgs3. Values represent the mean \pm s.d. from duplicate reactions. Assays were repeated three times. Student's $t$-test was used to calculate $p$ values $\left({ }^{\star \star} p<0.01\right)$. c Confocal images of third instar larval SGs from pgant9 ${ }^{R N A i}$ larvae that overexpress either pgant9A (pgant9RNAi, pgant9AOE) or pgant9B (pgant9 ${ }^{R N A i}$, pgant9B ${ }^{O E}$ ). All genotypes also express Sgs3-GFP (green). The white boxed area in the upper panels is enlarged below each image. Representative images from three independent experiments are shown. Scale bars, $10 \mu \mathrm{m}$ for upper panels and $2 \mu \mathrm{m}$ for lower panels. $\mathbf{d}$ Graphs of the cumulative frequency of granule circularity for each genotype are shown. Granule circularity quantification was performed on three individual SGs from three independent biological replicates. Cumulative frequency graph of pgant9RNAi, pgant9AOE ( $n=931$ granules) and pgant9RNAi, pgant9B ${ }^{O E}(n=946$ granules $)$ is overlapped with the data shown in Fig. $1 \mathrm{~b}$ for WT and CG30463 ${ }^{R N A i}$ (pgant9 ${ }^{R N A i}$ ). QPCR data to verify mRNA expression of pgant9A and pgant9B are shown in Supplementary Fig. 4a and western blots to verify protein expression are shown in Supplementary Fig. 4b, c

enough to the active site to be predicted to affect peptide recognition or access to the catalytic domain ${ }^{38-42}$ (Fig. 6). However, the structures presented herein show a unique a subunit loop that is longer and in closer proximity to the active site. Additionally, by solving the crystal structures of both splice variants, we provide the first evidence that splicing within the a subunit of the lectin domain serves as a molecular switch to change the charge of this extended loop, thus potentially modulating access to the active site within the catalytic domain. Moreover, we demonstrate in vitro that simply changing the charge of a peptide alters whether it is a preferred substrate for PGANT9A or PGANT9B, lending further support to a model where the charged a subunit modulates access of charged substrates to the active site of the enzyme. Many GTs contain substrate binding domains that are critical for their catalytic function ${ }^{33,50}$, but the lectin domain is unique to this family of glycosyltransferases. While the overall fold of the lectin domain is conserved, its orientation and primary sequence are variable throughout the family, resulting in a domain that fine tunes the substrate preference of each family member in a distinctive way, either by recognizing extant sugars on substrates or as we show in this study, by influencing peptide substrate preferences through electrostatic interactions. While our data support a model in which the charged lectin domain influences access of charged peptides to the active site of the catalytic domain, previous studies have suggested that certain lectin domains may also influence product release ${ }^{35}$. Future kinetic and binding studies will interrogate exactly how the unique lectin domains of PGANT9A and PGANT9B are influencing substrate specificity and catalytic efficiency. Regardless, the present study provides evidence that splicing can alter substrate specificity and that lectin domains have roles beyond GalNAc sugar recognition.

Our study also provides evidence that alternative splicing within pgant9 allows appropriate glycosylation of substrates unique to the salivary gland. Indeed PGANT9B, which contains the negatively charged a subunit and prefers to act on positively charged substrates, is the predominant splice variant in the salivary gland. Interestingly, the major cargos of the salivary gland consist of the mucin-like proteins Sgs3 and Eig72Ee, both of which contain positively charged regions that are predicted to be glycosylated. Another major secreted mucin produced by the salivary gland is Sgs ${ }^{51}$, which also contains positively charged regions that are predicted to be heavily glycosylated. This suggests that splicing within the a subdomain to generate PGANT9B may have evolved to ensure proper glycosylation of the positively charged substrates abundant within the salivary gland. Likewise, 
tissues that only express the PGANT9A variant (that prefers to act on negatively charged substrates) are those that have abundant expression of negatively charged mucins (e.g., Mur $18 B$ in the Malpighian tubules; http://flybase.org/reports/FBgn0030999). This study highlights a regulatory mechanism for modulating glycosyltransferase enzymatic specificity in vivo, according to the repertoire of substrates expressed in particular cells and tissues. Whether the expression and splicing of pgants is coordinated with substrate expression remains to be determined.

Whether other ppGalNAcT/PGANT family members are differentially spliced in vivo to generate alternative specificities remains to be investigated. While alternative splicing has been described for other ppGalNAcT family members ${ }^{52,53}$, no change in substrate specificity or sites of sugar addition were noted ${ }^{53}$. However, changes in substrate preferences may be challenging to detect in standard in vitro assays if the biologically relevant in vivo substrates are not known. Thus, many splicing events predicted from in silico analyses in other ppGalNAcT family members may confer unique specificities based on the tissues in which the splicing occurs and the predominant substrates expressed there. The additional degree of enzymatic variation conferred by splicing could make an already large family of enzymes (20 ppGalNAcTs in mammals and now 10 PGANTs in Drosophila $)^{29,52,54,55}$ even more functionally diverse and may serve to fine-tune enzymatic specificities based on the needs of the cell/tissue.

The factors that affect secretion, including cargo synthesis, modification, packaging and delivery to the extracellular environment are biologically relevant as disruptions in secretion or aberrations in the synthesis or integrity of cargo can contribute to diverse human diseases $s^{4-7,56,57}$. For example, secreted mucins are abundant along epithelial surfaces such as the digestive and respiratory tracts, where they mediate hydration and form a gellike layer that interacts with the microbiome and confers protection from infection and damage $e^{5,46,58,59}$. Many diseases of the digestive tract, such as colitis and colon cancer, have disruptions of the mucosal layer as one of their hallmarks ${ }^{56,57,60}$. Studies in model organisms have also highlighted a crucial role for the proper formation and secretion of this essential extracellular membrane $e^{4,6,59,61,62}$. As O-linked glycosylation is one of the

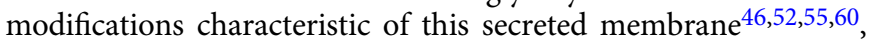
understanding how it is regulated at the enzymatic level as well as how the specific modifications influence protein structure, function, stability and/or bioavailability is essential. In this study, we demonstrate a regulatory paradigm for altering substrate selectivity among the enzymes that initiate O-glycosylation and demonstrate that altered O-glycosylation influences secretory granule morphology during maturation. Moreover, we demonstrate that expression of a particular splice variant (PGANT9B) that has specificity for the mucinous cargo of the salivary gland can restore secretory granule morphology, suggesting a key role for cargo glycosylation/structure in granule morphology. How this change in glycosylation specifically affects mucin structure during packaging and how it influences the biophysical properties of mucins once secreted will be the subject of future investigations. Moreover, understanding the role of this post-translational modification in secretory granule maturation and in mucin biology will aid in the development of mucin mimetics and strategies to treat diseases that are exacerbated by or the result of disruption of mucin biosynthesis and function.

\section{Methods}

CG30463/pgant9 cloning and DNA constructs used. DNA encoding pgant 9 (CG30463) was amplified by PCR using cDNA made from embryonic total RNA (Clontech), forward (5'-GCGGAATTCGATATGGCCTTCATCTGGCGG CGAC-3') and reverse (5'-CGCTCTAGACTCAACTTCGAGCTGTCGTAG-3') primers, and was cloned into the EcoRI and $\mathrm{XbaI}$ sites of the pIB/V5-His vector (Invitrogen). All primers used for cloning and PCR are listed in Supplementary Table 3. The pgant $9 A$ isoform was distinguishable from pgant $9 B$ isoform by NcoI digestion. For expression of the secreted form of pgant9A and pgant9B, DNA fragments without the $\mathrm{N}$-terminal cytoplasmic and transmembrane domains were amplified by PCR using pIB-pgant $9 A$ or pgant $9 B$ expression vector, forward ( $5^{\prime}$-CG CACGCGTACACGGATGACACCCGGC GT-3') and reverse (5'-CTTGCGGCCG CTCACAACTTCGAGCTGTCGTAG-3') primers, and then subcloned into MluI and Not I sites of pIMKF $4^{63}$ to generate FLAG-tagged, secreted proteins. cDNA coding Sgs3 and Sgs3-PTTTK were synthesized by GenScript (Piscataway, NJ) and subcloned into the EcoRI and NotI sites of pIB-V5His vector. For Sgs3- $\Delta$ or Sgs3T-Rich cloning, using pIB-Sgs3-V5His as template, PCR products were amplified by Sgs $3-\Delta$ forward (5'-TGGATGCCCCACCACACCTAAGCCGTG-3') and reverse (5'-GTGGTGGGGCATCCACAATCGCAACAGTTG-3'); or Sgs3-T-Rich forward (5'-CACAACACCCACCACACCTAAGCCGTG-3') and reverse (5'-GTG GTGGGTGTT GTGCACGGAAGTTGCG-3') primers. With the PCR products, the In-Fusion HD EcoDry Cloning Kit (TaKaRa) was used to synthesize the Sgs 3 deletions. For co-expression with Sgs3-V5 or Sgs3 deletions, V5-tag sequences of pIB-pgant $9 A$ and -pgant $9 B$ expression vectors were replaced with the FLAG Tag. Sequences of all plasmids were confirmed by DNA sequencing.

Fly stocks and genetics. All fly stocks and crosses were kept on MM media (KD Medical, Inc.) at $25^{\circ} \mathrm{C}$. The deficiency line used was $D f(2 R)$ Exel6064 (Bloomington \#7546). The c135-Gal4-driver line (Bloomington \#6978) was used to generate RNAi in the salivary gland. To visualize glue granules, the Sgs3-GFP line (Bloomington $\# 5885)^{20}$ was recombined with the c135-Gal4-driver line to generate Sgs3GFP, c135-Gal4 ${ }^{24}$. An RNAi target region for pgant9 was designed using Snapdragon (http://www.flyrnai.org/snapdragon) and off-targets were screened using a $19 \mathrm{bp}$-size threshold. The C-terminal region of pgant9 $(300 \mathrm{bp})$ was selected and amplified with the sense primer (5'-CGACGACTCCTGTCTCGATT-3') and the anti-sense primer (5'-GCTTGTGATTGTTGGTGTCG-3') and cloned into pWIZ vector as described previously to generate the UAS-pgant $9^{300 I R}$ vector ${ }^{30}$. For overexpression of pgant $9 A$ or pgant $9 B$ in the fly, the pUAST vector was used to generate UAS-pgant $9 A^{O E}$ or $U A S$-pgant $9 B^{O E}$. DNA fragments were amplified by PCR using pIB-pgant $9 A$ or pgant $9 B$ expression vector, forward $\left(5^{\prime}\right.$-GCGGAATT CGATATGGCCTTCATCTGGCGGCGAC-3') and reverse (5' - CTTGCGGCCG CTCACAACTTCGAGCTGTCGTAG-3') primers, and then subcloned into EcoRI and NotI sites of pUAST vector. All transgenic lines were generated by BestGene (Chino Hills, CA). The CG30463 ${ }^{\Delta}$ deletion strain (where exons 1-4 of CG30463 are deleted) was constructed through FRT- site-directed recombination using transposons $P\{X P\} d 11168$ (in the 5'UTR of CG30463/pgant9) and P\{Bac $\} C G 30463^{\text {f03628 }}$ (in the 4th intron of CG30463/pgant9) through genetic crosses of these lines as described previously ${ }^{64}$. Potential deletion mutants were screened by loss of miniwhite gene. Genomic DNA samples were obtained from these potential CG30463/ pgant9 mutants and used as templates for PCR amplification of a fragment encompassing the CG30463/pgant9 locus (forward primer, 5'-GTCATTCACAG GCTGAGAACAAC-3'; reverse primer, 5'-CTTAAGCCCAGCTACTATCTCAG G-3'). As predicted by the genome location of the transposons, we verified that the recombination generated a deletion of $41,681 \mathrm{bp}(2 \mathrm{R}: 16,689,713$ to $16,731,394)$ that exons 1-4 of CG30463/pgant9. Oregon $R$ stock was used for qPCR assessing gene expressing in wild type tissues in Fig. 3a. For wild type control, $w^{1118}$ (VDRC\#60000) stock was crossed with Sgs3-GFP, c135-Gal4 stock. The Sgs3-GFP line (Bloomington \#5884) ${ }^{20}$ was used as the WT control in Fig. 1c. For rescue experiments, the UAS-pgant $9^{R N A i}$ line (UAS-pgant9 $\left.300 I R \# 1\right)$ was recombined with

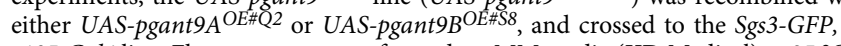
c135-Gal4 line. Fly crosses were performed on MM media (KD Medical) at $25^{\circ} \mathrm{C}^{30}$.

Salivary gland imaging and granule circularity measurements. Salivary glands from wandering third instar larvae were dissected in Schneider's medium and transferred to glass bottom dishes (MatTek) containing $50 \mu \mathrm{l}$ of media ${ }^{24}$. Samples were imaged on Nikon A1R+ confocal microscope with a Plan Apo IR X 60/1.27 numerical aperture (NA) water immersion (WI) objective. Imaging parameters for all samples such as wavelength, offset, detector, z-plane, zoom factor, and image resolution were kept constant. For granule circularity measurements, Nikon Imaging software (NIS-Elements AR version 4.5) was used to select individual granules that had clear outlines that were traceable by the software using the AutoDetect Binary tool. For analysis, 100 granules were selected by the AutoDetect. When the Autodetect outline aligned with the outer edge of the granule (within $\sim 1-2$ pixels) the granule was then added to the ROI (region of interest) list. After all of the granules were selected, the region of interest (ROI) was copied using the Binary toolbar and the measurement analysis tool was used to calculate the circularity of each selected granule. The software calculates circularity using the following formula: $(4 \times \pi \times \text { area/perimeter })^{2}$. A circularity value of 1 indicates a perfect circle while circularity values approaching 0 indicate an elongated polygon. Circularity analysis was performed on three independent biological replicates for each genotype. For each replicate, three glands were used for analysis and only three cells in each gland were used to measure a total of 800-950 individual granules per replicate. Values were exported to Microsoft Excel where the data was used to calculate frequency distributions for each genotype. The frequency bin was created with 0.5 intervals and data was normalized by dividing the frequency of the 
granules in the bin by the total number of granules analyzed. Values represent the mean \pm s.d. $P$-values were calculated using a two-tailed Student's $t$-test. No statistical method was used to predetermine sample size.

\section{Salivary gland dissection and Li-COR western blotting. PNA (Arachis hypogaea} lectin, Sigma-Aldrich L0881) was labeled with IRDye 800CW (Licor 928-38044). Salivary glands from wandering third instar larva were dissected in PBS and transferred to a $1.5 \mathrm{ml}$ eppendorf tube containing $50 \mu \mathrm{l}$ of RIPA buffer (Sigma) containing $1 \times$ Halt Protease Inhibitor (Thermo Scientific). Protein extracts from 3 glands was loaded in each lane of a NuPAGE 4-12\% Bis-Tris gel (Invitrogen). Gels were transferred onto nitrocellulose membranes. For Li-COR western blotting, the membranes were blocked with Odyssey Blocking Buffer (PBS-based) (Li-COR) and incubated with IRDye $800 \mathrm{CW}$-conjugated PNA (1:5000) overnight at $4{ }^{\circ} \mathrm{C}$. For the tubulin control blots, membranes were incubated with the tubulin antibody (1:1000) (Cell Signaling Technology, \#2125) overnight at $4{ }^{\circ} \mathrm{C}$, washed with PBS containing $0.1 \%$ Tween-20 (PBST) three times, and incubated with IRDye 680LTconjugated anti-rabbit IgG (1:10,000) (Li-COR, \#926-68021) for $1 \mathrm{~h}$ at room temperature. Membranes were washed with PBST three times, with PBS twice, and then scanned using a Li-COR Odyssey Infrared Imaging System. Full-length western blots are shown in Supplementary Fig. 5.

S2R + cell culture, immunostaining and western blotting. Drosophila S2R + cells (Drosophila Genomics Resource Center, \#150) were cultured in Schneider's medium (Invitrogen) containing 10\% heat-inactivated fetal bovine serum (Invitrogen) at $25^{\circ} \mathrm{C}$. For immunostaining, S2R + cells were grown on cover glass and transfected with plasmids using Effectene transfection reagent (Qiagen) according to the manufacturer's instructions. After 3 days, cells were fixed with $4 \%$ formaldehyde in phosphate buffered saline (PBS) and stained with the monoclonal Anti-V5-Cy $3^{\text {Ts }}$ antibody (1:300) (Sigma, \#V4014), the GM130 antibody (1:100) (Abcam, \#ab30637), and Alexa Fluor ${ }^{\circledR} 647$ AffiniPure F(ab') ${ }_{2}$ Fragment Donkey Anti-Rabbit $\operatorname{IgG}(\mathrm{H}+\mathrm{L})$ (1:200) (Jackson Immunoresearch, \#711-606-152), each at room temperature for $1 \mathrm{~h}$ as described previously ${ }^{65}$. Samples were imaged on a Nikon A1R + confocal microscope with a CFI Plan Apochromat Lambda X 60/1.4 numerical aperture (NA) oil immersion objective. For western blotting, S2R + cells were grown on a 12-well plate and transfected with plasmids. After 3 days, cells were collected and lysed with RIPA buffer containing 1X Halt Protease Inhibitor (Thermo Scientific). Protein extracts were resolved using NuPAGE 4-12\% Bis-Tris gels (Invitrogen) and transferred onto nitrocellulose membranes. For lectin blots, the membranes were blocked by 1X blocking buffer (Sigma, \#B6429) and incubated with HRP-conjugated HPA lectin (1:1000) (EY Laboratories, Inc., \#H-3601-1) diluted in blocking buffer overnight at $4{ }^{\circ} \mathrm{C}$. After washing three times with PBS containing $0.1 \%$ Tween-20 (PBST), specific bands were visualized using enhanced chemiluminescence and analyzed by LAS 3000 Imaging System (Fujifilm). For immunoblotting, anti-V5-HRP (1:5000) (Invitrogen, \#R961-25), anti-Tubulin (1:1000) (Cell Signaling Technology, \#2125), and HRP-conjugated anti-rabbit antibody (1:2000) (Cell Signaling Technology, \#7074) were used. For immunoprecipitation, $10 \mu \mathrm{l}$ of agarose-conjugated V5 antibody (Sigma, \#A7345) was incubated with cell lysates for $2 \mathrm{~h}$ at room temperature. Beads were washed with PBST four times and protein samples were eluted by LDS sample buffer (Invi trogen, \#NP0007) containing $\beta$-mercaptoethanol. For Licor Western blotting, HPA (Helix pomatia lectin, Sigma-Aldrich L3382) were labeled with IRDye 800CW (Licor 928-38044). The membranes were blocked with Odyssey Blocking Buffer (PBS-based) (Li-COR) and incubated with IRDye 800CW-conjugated HPA $(1: 10,000)$ and anti-V5 antibody (1:5000) (Invitrogen, \#R96025) overnight at $4{ }^{\circ} \mathrm{C}$. Then the membranes were washed with PBS containing $0.1 \%$ Tween-20 (PBST) three times, and incubated with IRDye 680LT-conjugated anti-mouse IgG $(1: 10,000)$ (Li-COR, \# 926-68020) for $1 \mathrm{~h}$ at room temperature. After wash, membranes were scanned using a Li-COR Odyssey Infrared Imaging System.The cell lines used tested negative to mycoplasma contamination.

GalNAc transferase assays. Peptide substrates were synthesized by AnaSpec (Fremont, CA). Enzyme activity assays were performed as described previously ${ }^{29}$. Briefly, enzyme assays were performed using equal amounts of purified PGANT9A and PGANT9B. Each reaction was performed in triplicate at $37^{\circ} \mathrm{C}$ for $1 \mathrm{~h}$. Reac tions were performed in $25 \mu \mathrm{l}$ final volumes consisting of the following: $500 \mu \mathrm{M}$ acceptor substrate, $7.3 \mu \mathrm{M}{ }^{14} \mathrm{C}$-UDP-GalNAc $(54.7 \mathrm{mCi} / \mathrm{mmol} ; 0.02 \mathrm{mCi} / \mathrm{ml}), 44$ $\mu \mathrm{M}$ cold UDP-GalNAc, $10 \mathrm{mM} \mathrm{MnCl}_{2}, 40 \mathrm{mM}$ cacodylate ( $\mathrm{pH} 6.5$ ), $40 \mathrm{mM} 2$ mercaptoethanol and $0.1 \%$ Triton X-100. Reaction products were purified using anion exchange chromatography (AG $1 \times-8$, Bio-Rad). Reactions without acceptor peptide were used to generate background values that were subtracted from each experimental value. Adjusted experimental values for each substrate were then averaged and standard deviations were calculated. Enzyme activity (initial rate) is expressed as $\mathrm{dpm} / \mathrm{h}$.

Quantitative RT-PCR. Quantitative RT-PCR (qPCR) was performed using the PCR primers designed using Beacon Designer software (BioRad) (pgant9A Sense: 5'-ATGGTATCTGGACAACATT-3', Anti-sense: 5'-CTTCTTCTGGTGC

TTCTT-3'; pgant9B Sense: 5'-AATGGTATCTGGACAACA-3', Anti-sense: 5'-GG CACTCATAAATGGAAA-3'; 18S rRNA Sense: 5' - GGACACGCAAACTTCTCA
ACAGC-3', Anti-sense: 5'-AATCTTCAGAGCCAATCCTTATCCC-3'). For tissue-specific expression of pgant9 isoforms, third instar wandering larvae (Oregon R) were dissected in PBS and tissues were collected according to FlyAtlas guidelines (http://flyatlas.org/about_atlas.html). For RNAi efficiency and isoform specific overexpression, salivary glands from wandering third instar larvae were dissected in PBS and collected. RNA was isolated using RNAqeous Micro Total RNA kit (Invitrogen) and cDNA synthesis was performed using iScript cDNA Synthesis Kit (Bio-Rad). qPCR was performed on a CFX96 real time PCR thermocycler (Bio-Rad) using the SYBR-Green PCR Master Mix (Bio-Rad). RNA levels were normalized to $18 \mathrm{~S}$ rRNA. Values represent the mean \pm s.d. $P$-values were calculated using two-tailed Student's $t$-test.

Mass spectrometry. Salivary gland dissection, protein extraction and gel electrophoresis were carried out as described above. Gels were stained with Simply Blue Safe Stain (Invitrogen) using the protocol as suggested by the manufacturer. The three bands of interest were cut and sent for analysis to Poochon Scientific. The LC/MS/MS analysis was carried out using a Thermo Scientific Q-Exactive hybrid Quadrupole-Orbitrap Mass Spectrometer and a Thermo Dionex UltiMate 3000 RSLCnano System. Peptides from each sample were loaded onto a peptide trap cartridge at a flow rate of $5 \mu \mathrm{l} / \mathrm{min}$. The trapped peptides were eluted onto a reversed-phase PicoFrit column (New Objective, Woburn, MA) using a linear gradient of acetonitrile (3-36\%) in $0.1 \%$ formic acid. The elution duration was $60 \mathrm{~min}$ per fraction at a flow rate of $0.3 \mu \mathrm{l} / \mathrm{min}$. Eluted peptides from the PicoFrit column were ionized and sprayed into the mass spectrometer, using a Nanospray Flex Ion Source ES071 (Thermo) under the following settings: spray voltage, 1.6 $\mathrm{kV}$, Capillary temperature, $250^{\circ} \mathrm{C}$. Other settings were empirically determined. Raw data file was searched against public NCBI database of Drosophila melanogaster using the Proteome Discoverer 1.4 software (Thermo, San Jose, CA) based on the SEQUEST algorithm. Carbamidomethylation $(+57.021 \mathrm{Da})$ of cysteines was fixed modification, and Oxidation and Deamidation Q/N-deamidated $(+0.98402$ $\mathrm{Da}), \mathrm{S} / \mathrm{T}$ O-GalNAc $(+203.079 \mathrm{Da})$ were set as dynamic modifications. The minimum peptide length was specified to be five amino acids. The precursor mass tolerance was set to $15 \mathrm{ppm}$, whereas fragment mass tolerance was set to $0.05 \mathrm{Da}$. The maximum false peptide discovery rate was specified as 0.01 . The resulting Proteome Discoverer Report contains all assembled proteins with peptides sequences and peptide spectrum match counts (PSM\#).

Protein preparation. PGANT9A (AA 146-650) and PGANT9B (AA 146-647) were cloned into the Pichia pastoris expression vector pPICZa A (Invitrogen) between the EcoRI and XbaI cut sites to generate His ${ }_{6}$-TEV-Gly 4 -PGANT9 constructs. The expression vectors were linearized with PmeI and transformed into SMD1168 (Invitrogen) cells by electroporation to generate stable PGANT9A and PGANT9B $P$. pastoris strains. Cells expressing PGANT9A or PGANT9B were grown in BMGY media, $\mathrm{pH} 6.0$ in the presence of $100 \mu \mathrm{g} / \mathrm{ml}$ of Zeocin (Invivogen) at $30^{\circ} \mathrm{C}$ to an $\mathrm{OD}_{600} \sim 10.0$. To induce expression, cells were centrifuged at $1500 \times g$ for $15 \mathrm{~min}$ and resuspended in BMMY media, $\mathrm{pH} 6.0$ in the presence of $100 \mu \mathrm{g} / \mathrm{ml}$ of Zeocin and grown at $20^{\circ} \mathrm{C}$ for $24 \mathrm{hrs}$. The supernatant was collected by centrifugation at $1500 \times g$ for $15 \mathrm{~min}$ and further cleared by filtration, followed by the addition of $50 \mathrm{mM}$ Tris $\mathrm{pH} 7.5$ and $10 \mathrm{mM} \beta \mathrm{ME}$. All subsequent purification steps were carried out at $4{ }^{\circ} \mathrm{C}$. The supernatant was loaded onto a $5 \mathrm{ml}$ HisTrap HP (GE Healthcare) column equilibrated with buffer (A) containing $25 \mathrm{mM}$ Tris, $250 \mathrm{mM}$ $\mathrm{NaCl}, 10 \mathrm{mM} \beta \mathrm{ME}, \mathrm{pH}$ 7.5. The column was then washed with 10 Column Volumes $(\mathrm{CV})$ of buffer $\mathrm{A}$ and the protein was eluted using a linear gradient of 30-300 $\mathrm{mM}$ imidazole over $10 \mathrm{CV}$. Peak fractions were pooled and dialyzed overnight in buffer A with $25 \mathrm{mM}$ imidazole at $4{ }^{\circ} \mathrm{C}$ in the presence of $1.5 \mathrm{mg}$ of $\mathrm{His}_{6}$-TEV protease to cleave the $\mathrm{His}_{6}$-Tag. The sample was passed through a $1 \mathrm{ml}$ HisTrap HP (GE Healthcare) column to separate the pure PGANT9 from the $\mathrm{His}_{6}$ Tag and $\mathrm{His}_{6}$-TEV. Glycerol was then added to the tag-free PGANT9 to a final concentration of $20 \%$ and the protein was frozen in $\mathrm{LN}_{2}$ before storing at $-80^{\circ} \mathrm{C}$.

High mannose chains were cleaved before preparing the samples for crystallization. Purified enzyme was first thawed and incubated with 50,000 units of MBP-tagged Endoglycosidase H (Endo Hf, New England Biolabs) per mg of enzyme and incubated overnight at $4{ }^{\circ} \mathrm{C}$. The PGANT9 was separated from the Endo $\mathrm{Hf}$ on $2 \mathrm{ml}$ of amylose resin (New England Biolabs) per mg of enzyme equilibrated with buffer (B) containing $100 \mathrm{mM} \mathrm{NaCl}, 20 \mathrm{mM}$ HEPES, $0.5 \mathrm{mM}$ EDTA, $10 \mathrm{mM} \beta \mathrm{ME}, \mathrm{pH} 7.3$. The resin was washed with $2 \mathrm{CV}$ of buffer $\mathrm{B}$ and the flow-through and wash containing PGANT9 were pooled. The protein sample was concentrated in a $30,000 \mathrm{kDa}$ cutoff centrifugal filter (Amicon, Millipore) to $100 \mu \mathrm{l}$ and buffer exchanged by adding $4 \mathrm{ml}$ of buffer $\mathrm{B}$ and concentrating to $100 \mu \mathrm{l}$. To obtain the final sample for crystallization studies, $4 \mathrm{ml}$ of buffer B were added a second time, and the protein was concentrated to $10 \mathrm{mg} / \mathrm{ml}$. The activity of the $P$. pastoris purified enzymes against the Sgs 3 substrates was verified as described in above.

Crystallization, data collection and structure refinement. The PGANT9peptide-UDP-Mn ${ }^{2+}$ complex was prepared by combining PGANT9A or PGANT9B with $5 \mathrm{mM}$ peptide substrate (PTTTE for PGANT9A and PTTTK for PGANT9B (Anaspec, Fremont, CA), see Fig. 7a), $5 \mathrm{mM} \mathrm{UDP}$, and $5 \mathrm{mM} \mathrm{MnCl}_{2}$ to a final protein concentration of $6 \mathrm{mg} / \mathrm{ml}$, and the complex was incubated at RT fo 
30 min before placing on ice. Crystallization was initiated by mixing $1 \mu \mathrm{l}$ of complex with $1 \mu \mathrm{l}$ of well buffer using the hanging drop vapor diffusion method. PGANT9A crystals grew as $200 \mu \mathrm{M} \times 200 \mu \mathrm{M}$ square-shaped plates in $22 \%$ PEG3350, $0.2 \mathrm{M}$ sodium malonate, and $20 \mathrm{mM}$ strontium chloride hexahydrate. PGANT9B crystals grew as $300 \mu \mathrm{M} \times 50 \mu \mathrm{M}$ rods in $20 \%$ PEG3350 and $0.18 \mathrm{M}$ ammonium citrate. Crystals of PGANT9A and PGANT9B were cryo-protected with well buffer containing $20 \%$ glycerol and flash frozen in liquid nitrogen. X-ray data was collected at the Advanced Photon Source using the SER-CAT BM- and ID-22 beam lines (Argonne, IL) 360 degrees of data were collected for PGANT9A and 300 degrees of data were collected for PGANT9B. Both data sets were collected at the wavelength of $1.0 \AA$ and processed and scaled using HKL2000 ${ }^{66}$. The structure of PGANT9B was solved by molecular replacement using MolRep (CCP4i) ${ }^{67,68}$ and human GalNAc-T1 as a search model (PDB: 1XHB). The lectin domain was manually rebuilt in $\operatorname{Coot}^{69}$ and the structure of PGANT9B was used for solving the structure of PGANT9A by molecular replacement (MolRep, CCP4i). The structures of PGANT9A and PGANT9B were refined in PHENIX ${ }^{70}$ to 2.8 and $2.07 \AA$ resolution, respectively (Table 1). For PGANT9A, 97\% of residues are in the favored region of the Ramachandran plot, $2.6 \%$ are allowed, and $0.15 \%$ are outliers. For PGANT9B, $97.3 \%$ of residues are in the favored region of the Ramachandran plot, $2.7 \%$ are allowed, and $0 \%$ are outliers. Figures and electrostatic potential maps were generated in Pymol (The PyMOL Molecular Graphics System, Version 2.0 Schrodinger, LLC).

Statistical analyses. Number of replicates used for each analysis is specified in the figure legends. For granule morphology, every granule analyzed was used. $P$-values were calculated using the two-tailed Student's $t$-test. No statistical method was used to predetermine sample size, no randomization methods were used for these studies and no blinding studies were performed.

Data availability. Data supporting the findings of this manuscript are available from the corresponding author upon reasonable request. The coordinates and structure factors have been deposited in the Protein Data Bank under the accession codes: PDB 6E4Q (PGANT9A) and PDB 6E4R (PGANT9B). The mass spectrometry proteomics data have been deposited to the ProteomeXchange Consortium via the PRIDE partner repository with accession numbers PXD010548 (band 1) and PDX010549 (band 3).

Received: 9 February 2018 Accepted: 1 August 2018

Published online: 29 August 2018

\section{References}

1. Burgoyne, R. D. \& Morgan, A. Secretory granule exocytosis. Physiol. Rev. 83, 581-632 (2003).

2. Porat-Shliom, N., Milberg, O., Masedunskas, A. \& Weigert, R. Multiple roles for the actin cytoskeleton during regulated exocytosis. Cell Mol. Life Sci. 70, 2099-2121 (2013).

3. Stockli, J., Fazakerley, D. J. \& James, D. E. GLUT4 exocytosis. J. Cell Sci. 124, 4147-4159 (2011).

4. Bergstrom, K. et al. Defective intestinal mucin-type O-glycosylation causes spontaneous colitis-associated cancer in mice. Gastroenterology 151, 152-164 e111 (2016).

5. Johansson, M. E. et al. Bacteria penetrate the normally impenetrable inner colon mucus layer in both murine colitis models and patients with ulcerative colitis. Gut 63, 281-291 (2014).

6. Velcich, A. et al. Colorectal cancer in mice genetically deficient in the mucin Muc2. Science 295, 1726-1729 (2002).

7. Liu, J., Walker, N. M., Ootani, A., Strubberg, A. M. \& Clarke, L. L. Defective goblet cell exocytosis contributes to murine cystic fibrosis-associated intestinal disease. J. Clin. Invest. 125, 1056-1068 (2015).

8. Rutter, G. A., Varadi, A., Tsuboi, T., Parton, L. \& Ravier, M. Insulin secretion in health and disease: genomics, proteomics and single vesicle dynamics. Biochem. Soc. Trans. 34, 247-250 (2006).

9. Barlowe, C. K. \& Miller, E. A. Secretory protein biogenesis and traffic in the early secretory pathway. Genetics 193, 383-410 (2013).

10. $\mathrm{Du}, \mathrm{W}$. et al. HID-1 is required for homotypic fusion of immature secretory granules during maturation. eLife 5, e18134 (2016).

11. Orci, L. et al. Proteolytic maturation of insulin is a post-Golgi event which occurs in acidifying clathrin-coated secretory vesicles. Cell 49, 865-868 (1987).

12. Osborne, S. L., Herreros, J., Bastiaens, P. I. \& Schiavo, G. Calcium-dependent oligomerization of synaptotagmins I and II. Synaptotagmins I and II are localized on the same synaptic vesicle and heterodimerize in the presence of calcium. J. Biol. Chem. 274, 59-66 (1999).

13. Tooze, S. A., Martens, G. J. \& Huttner, W. B. Secretory granule biogenesis: rafting to the SNARE. Trends Cell Biol. 11, 116-122 (2001).
14. Tran, D. T. \& Ten Hagen, K. G. Real-time insights into regulated exocytosis. J. Cell Sci. 130, 1355-1363 (2017).

15. Urbe, S., Dittie, A. S. \& Tooze, S. A. pH-dependent processing of secretogranin II by the endopeptidase PC2 in isolated immature secretory granules. Biochem. J. 321(Pt 1), 65-74 (1997)

16. Wendler, F., Page, L., Urbe, S. \& Tooze, S. A. Homotypic fusion of immature secretory granules during maturation requires syntaxin 6. Mol. Biol. Cell 12, 1699-1709 (2001).

17. Ambort, D. et al. Calcium and $\mathrm{pH}$-dependent packing and release of the gelforming MUC2 mucin. Proc. Natl Acad. Sci. USA 109, 5645-5650 (2012).

18. Korge, G. Larval saliva in Drosophila melanogaster: production, composition, and relationship to chromosome puffs. Dev. Biol. 58, 339-355 (1977).

19. Lehmann, M. Drosophila Sgs genes: stage and tissue specificity of hormone responsiveness. Bioessays 18, 47-54 (1996).

20. Biyasheva, A., Do, T. V., Lu, Y., Vaskova, M. \& Andres, A. J. Glue secretion in the Drosophila salivary gland: a model for steroid-regulated exocytosis. Dev. Biol. 231, 234-251 (2001)

21. Burgess, J. et al. AP-1 and clathrin are essential for secretory granule biogenesis in Drosophila. Mol. Biol. Cell 22, 2094-2105 (2011).

22. Farkas, R. \& Suakova, G. Developmental regulation of granule size and numbers in larval salivary glands of Drosophila by steroid hormone ecdysone. Cell Biol. Int. 23, 671-676 (1999).

23. Burgess, J. et al. Type II phosphatidylinositol 4-kinase regulates trafficking of secretory granule proteins in Drosophila. Development 139, 3040-3050 (2012).

24. Tran, D. T., Masedunskas, A., Weigert, R. \& Ten Hagen, K. G. Arp2/3mediated F-actin formation controls regulated exocytosis in vivo. Nat. Commun. 6, 10098 (2015).

25. Rousso, T., Schejter, E. D. \& Shilo, B. Z. Orchestrated content release from Drosophila glue-protein vesicles by a contractile actomyosin network. Nat. Cell Biol. 18, 181-190 (2016).

26. Segal, D., Zaritsky, A., Schejter, E. D. \& Shilo, B. Z. Feedback inhibition of actin on Rho mediates content release from large secretory vesicles. J. Cell Biol. 217, 1815-1826 (2018)

27. Zhang, L. et al. O-glycosylation regulates polarized secretion by modulating Tangol stability. Proc. Natl Acad. Sci. USA 111, 7296-7301 (2014).

28. Schwientek, T. et al. Functional conservation of subfamilies of putative UDP$\mathrm{N}$-acetylgalactosamine:polypeptide $\mathrm{N}$-acetylgalactosaminyltransferases in Drosophila, Caenorhabditis elegans, and mammals. One subfamily composed of l(2)35Aa is essential in Drosophila. J. Biol. Chem. 277, 22623-22638 (2002).

29. Ten Hagen, K. G., Tran, D. T., Gerken, T. A., Stein, D. S. \& Zhang, Z. Functional characterization and expression analysis of members of the UDPGalNAc:polypeptide $N$-acetylgalactosaminyltransferase family from Drosophila melanogaster. J. Biol. Chem. 278, 35039-35048 (2003).

30. Tran, D. T. et al. Multiple members of the UDP-GalNAc: polypeptide $N$ acetylgalactosaminyltransferase family are essential for viability in Drosophila. J. Biol. Chem. 287, 5243-5252 (2012).

31. Lombard, V., Golaconda Ramulu, H., Drula, E., Coutinho, P. M. \& Henrissat, B. The carbohydrate-active enzymes database (CAZy) in 2013. Nucleic Acids Res. 42, D490-D495 (2014).

32. Hurtado-Guerrero, R. \& Davies, G. J. Recent structural and mechanistic insights into post-translational enzymatic glycosylation. Curr. Opin. Chem. Biol. 16, 479-487 (2012).

33. Gloster, T. M. Advances in understanding glycosyltransferases from a structural perspective. Curr. Opin. Struct. Biol. 28, 131-141 (2014).

34. Gerken, T. A. et al. The lectin domain of the polypeptide GalNAc transferase family of glycosyltransferases (ppGalNAc Ts) acts as a switch directing glycopeptide substrate glycosylation in an N- or C-terminal direction, further controlling mucin type O-glycosylation. J. Biol. Chem. 288, 19900-19914 (2013).

35. Raman, J. et al. The catalytic and lectin domains of UDP-GalNAc:polypeptide alpha- $\mathrm{N}$-Acetylgalactosaminyltransferase function in concert to direct glycosylation site selection. J. Biol. Chem. 283, 22942-22951 (2008).

36. Aoki, K. et al. The diversity of O-linked glycans expressed during Drosophila melanogaster development reflects stage- and tissue-specific requirements for cell signaling. J. Biol. Chem. 283, 30385-30400 (2008).

37. Korayem, A. M. et al. A Drosophila salivary gland mucin is also expressed in immune tissues: evidence for a function in coagulation and the entrapment of bacteria. Insect Biochem. Mol. Biol. 34, 1297-1304 (2004).

38. Fritz, T. A., Hurley, J. H., Trinh, L. B., Shiloach, J. \& Tabak, L. A. The beginnings of mucin biosynthesis: the crystal structure of UDP-GalNAc: polypeptide alpha- $\mathrm{N}$-acetylgalactosaminyltransferase-T1. Proc. Natl Acad. Sci. USA 101, 15307-15312 (2004).

39. Fritz, T. A., Raman, J. \& Tabak, L. A. Dynamic association between the catalytic and lectin domains of human UDP-GalNAc:polypeptide alpha- $N$ acetylgalactosaminyltransferase-2. J. Biol. Chem. 281, 8613-8619 (2006).

40. Kubota, T. et al. Structural basis of carbohydrate transfer activity by human UDP-GalNAc: polypeptide alpha- $N$-acetylgalactosaminyltransferase (ppGalNAc-T10). J. Mol. Biol. 359, 708-727 (2006). 
41. Lira-Navarrete, E. et al. Dynamic interplay between catalytic and lectin domains of GalNAc-transferases modulates protein O-glycosylation. Nat. Commun. 6, 6937 (2015).

42. Rivas, M. L. et al. The interdomain flexible linker of the polypeptide GalNAc transferases dictates their long-range glycosylation preferences. Nat. Commun. 8, 1959 (2017).

43. Lira-Navarrete, E. et al. Substrate-guided front-face reaction revealed by combined structural snapshots and metadynamics for the polypeptide $\mathrm{N}$ acetylgalactosaminyltransferase 2. Angew. Chem. Int. Ed. Engl. 53, 8206-8210 (2014).

44. Coltart, D. M. et al. Principles of mucin architecture: structural studies on synthetic glycopeptides bearing clustered mono-, di-, tri-, and hexasaccharide glycodomains. J. Am. Chem. Soc. 124, 9833-9844 (2002).

45. Shogren, R., Gerken, T. A. \& Jentoft, N. Role of glycosylation on the conformation and chain dimensions of O-linked glycoproteins: light-scattering studies of ovine submaxillary mucin. Biochemistry 28, 5525-5536 (1989).

46. Tabak, L. A. In defense of the oral cavity: structure, biosynthesis, and function of salivary mucins. Annu. Rev. Physiol. 57, 547-564 (1995).

47. Chaffey, P. K. et al. Structural insight into the stabilizing effect of Oglycosylation. Biochemistry 56, 2897-2906 (2017).

48. Godl, K. et al. The $\mathrm{N}$ terminus of the MUC2 mucin forms trimers that are held together within a trypsin-resistant core fragment. J. Biol. Chem. 277, 47248-47256 (2002).

49. Lidell, M. E. et al. The recombinant C-terminus of the human MUC2 mucin forms dimers in Chinese-hamster ovary cells and heterodimers with fulllength MUC2 in LS 174T cells. Biochem. J. 372, 335-345 (2003).

50. Zhang, $H$. et al. New helical binding domain mediates a glycosyltransferase activity of a bifunctional protein. J. Biol. Chem. 291, 22106-22117 (2016).

51. Velissariou, V. \& Ashburner, M. The secretory proteins of the larval salivary gland of Drosophila melanogaster: cytogenetic correlation of a protein and a puff. Chromosoma 77, 13-27 (1980).

52. Bennett, E. P. et al. Control of mucin-type O-glycosylation: a classification of the polypeptide GalNAc-transferase gene family. Glycobiology 22, 736-756 (2012).

53. Raman, J., Guan, Y., Perrine, C. L., Gerken, T. A. \& Tabak, L. A. UDP-Nacetyl-alpha-D-galactosamine:polypeptide $\mathrm{N}$-acetylgalactosaminyltransferases: completion of the family tree. Glycobiology 22, 768-777 (2012).

54. Ten Hagen, K. G., Zhang, L., Tian, E. \& Zhang, Y. Glycobiology on the fly: developmental and mechanistic insights from Drosophila. Glycobiology 19, 102-111 (2009).

55. Tran, D. T. \& Ten Hagen, K. G. Mucin-type O-glycosylation during development. J. Biol. Chem. 288, 6921-6929 (2013).

56. Guda, K. et al. Inactivating germ-line and somatic mutations in polypeptide $\mathrm{N}$-acetylgalactosaminyltransferase 12 in human colon cancers. Proc. Natl Acad. Sci. USA 106, 12921-12925 (2009).

57. Larsson, J. M. et al. Altered O-glycosylation profile of MUC2 mucin occurs in active ulcerative colitis and is associated with increased inflammation. Inflamm. Bowel Dis. 17, 2299-2307 (2011).

58. Tabak, L. A., Levine, M. J., Mandel, I. D. \& Ellison, S. A. Role of salivary mucins in the protection of the oral cavity. J. Oral Pathol. 11, 1-17 (1982).

59. Zhang, L., Turner, B., Ribbeck, K. \& Ten Hagen, K. G. Loss of the mucosal barrier alters the progenitor cell niche via Janus kinase/signal transducer and activator of transcription (JAK/STAT) signaling. J. Biol. Chem. 292, 21231-21242 (2017).

60. Johansson, M. E. \& Hansson, G. C. Immunological aspects of intestinal mucus and mucins. Nat. Rev. Immunol. 16, 639-649 (2016).

61. Gao, N. et al. Loss of intestinal O-glycans promotes spontaneous duodenal tumors. Am. J. Physiol. Gastrointest. Liver Physiol. 311, G74-G83 (2016).

62. Van der Sluis, M. et al. Muc2-deficient mice spontaneously develop colitis, indicating that MUC2 is critical for colonic protection. Gastroenterology 131, 117-129 (2006).

63. Ten Hagen, K. G. \& Tran, D. T. A UDP-GalNAc:polypeptide Nacetylgalactosaminyltransferase is essential for viability in Drosophila melanogaster. J. Biol. Chem. 277, 22616-22622 (2002).
64. Parks, A. L. et al. Systematic generation of high-resolution deletion coverage of the Drosophila melanogaster genome. Nat. Genet. 36, 288-292 (2004).

65. Zhang, L. \& Ten Hagen, K. G. Dissecting the biological role of mucin-type Oglycosylation using RNA interference in Drosophila cell culture. J. Biol. Chem. 285, 34477-34484 (2010).

66. Otwinowski, Z. \& Minor, W. Processing of X-ray diffraction data collected in oscillation mode. Methods Enzymol. 276, 307-326 (1997).

67. Potterton, E., Briggs, P., Turkenburg, M. \& Dodson, E. A graphical user interface to the CCP4 program suite. Acta Crystallogr. D 59, 1131-1137 (2003).

68. Winn, M. D. et al. Overview of the CCP4 suite and current developments. Acta Crystallogr. D 67, 235-242 (2011).

69. Emsley, P., Lohkamp, B., Scott, W. G. \& Cowtan, K. Features and development of Coot. Acta Crystallogr. D 66, 486-501 (2010).

70. Adams, P. D. et al. PHENIX: a comprehensive Python-based system for macromolecular structure solution. Acta Crystallogr. D 66, 213-221 (2010).

\section{Acknowledgements}

We would like to sincerely thank our colleagues for many helpful discussions. We also thank the Bloomington Stock Center, the Developmental Studies Hybridoma Bank and the Vienna Drosophila RNAi Center for providing fly stocks, antibodies and other reagents. Mass spectrometry was performed by Poochon Scientific (Frederick, MD). This research was supported by the Intramural Research Program of the NIDCR at the National Institutes of Health (Z01-DE-000713 to K.G.T.H. and 1-ZIA-DE000739-05 to L.A.T.).

\section{Author contributions}

S.J., N.S., L.R., L.Z. and D.T.T., L.A.T. and K.G.T.H. designed and planned the research. S.J., N.S., L.R., L.Z., K.M. and D.T.T. performed the experiments. N.S. solved the crystal structures. S.J., N.S., L.R., L.Z., D.T.T., L.A.T. and K.G.T.H. analyzed and discussed the data. K.G.T.H. wrote the paper with assistance from S.J., N.S., L.R., L.Z., D.T.T., L.A.T.

\section{Additional information}

Supplementary Information accompanies this paper at https://doi.org/10.1038/s41467018-05978-9.

Competing interests: The authors declare no competing interests.

Reprints and permission information is available online at http://npg.nature.com/ reprintsandpermissions/

Publisher's note: Springer Nature remains neutral with regard to jurisdictional claims in published maps and institutional affiliations.

Open Access This article is licensed under a Creative Commons Attribution 4.0 International License, which permits use, sharing, adaptation, distribution and reproduction in any medium or format, as long as you give appropriate credit to the original author(s) and the source, provide a link to the Creative Commons license, and indicate if changes were made. The images or other third party material in this article are included in the article's Creative Commons license, unless indicated otherwise in a credit line to the material. If material is not included in the article's Creative Commons license and your intended use is not permitted by statutory regulation or exceeds the permitted use, you will need to obtain permission directly from the copyright holder. To view a copy of this license, visit http://creativecommons.org/ licenses/by/4.0/

(c) The Author(s) 2018 